\title{
Tail Dependence and Heavy Tailedness in Extreme Risks
}

\author{
by \\ Liuyan Ji \\ A thesis \\ presented to the University of Waterloo \\ in fulfillment of the \\ thesis requirement for the degree of \\ Master of Mathematics \\ in \\ Statistics
}

Waterloo, Ontario, Canada, 2020

(C) Liuyan Ji 2020 


\section{Author's Declaration}

I hereby declare that I am the sole author of this thesis. This is a true copy of the thesis, including any required final revisions, as accepted by my examiners.

I understand that my thesis may be made electronically available to the public. 


\begin{abstract}
In the modelling of multivariate extreme risks, the tail dependence and the heavy tailedness are the two key factors. Heavy tailedness are usually defined through the regular variation. Tail dependence can be modelled by copulas with the so-called tail order property. In this thesis, we first investigate important characteristics of the copulas with tail order property. Then, as an alternative of quantifying extreme risks, we propose a new risk measure called the Joint Expected Shortfall (JES). The JES, which can be viewed as a consolidation of both Expected Shortfall (ES) and Marginal Expected Shortfall (MES) risk measures, has the desirable property of measuring risk by jointly capturing both tail dependence and heavy tailedness. The asymptotic analysis of JES is conducted to provide a simple and transparent way of studying the interplay between tail dependence and heavy tailedness. Various examples are presented to illuminate our results. In particular, risk measures such as ES and MES that ignore the joint effect of dependence and heavy tailedness may severely underestimate the underlying risk.
\end{abstract}




\section{Acknowledgements}

First, I express my deepest gratitudes to my research supervisors, Professor Kenseng Tan and Professor Fan Yang, for providing invaluable guidance throughout my graduate study.

Professor Tan's ingenuousness and huge passion to his career set an example for me, and it always inspires me. Without his phenomenal supports, I would not be able to finish the thesis. Professor Yang introduced me the interesting research topic and always gave me sincerest suggestions when challenge occurred. She provided me tremendous help both academically and emotionally.

I would like to express my thankfulness to all the faculty members of the Department of Statistics and Actuarial Science. I would like to thank Professors who taught and guided me during my graduate study: Dr. Adam Kolkiewicz, David Landriault, Dr. Christiane Lemieux, Dr. David Saunders, Dr. Alexander Schied, Dr. Ruodu Wang, Dr. Chengguo Weng, Dr Tony Wirjanto, Dr. Changbao Wu and Dr. Fan Yang. I also want to thank Ms. Mary Lou Dufton and Ms. Lisa Baxter for their kind and generous help.

Finally, I am extremely grateful to my parents for their endless supports, and I am greatly indebted to my friends for their companionship and encouragement. 


\section{Dedication}

In memory of my beloved grandma. 


\section{Table of Contents}

List of Figures $\quad$ viii

$\begin{array}{ll}\text { List of Tables } & \text { ix }\end{array}$

1 Introduction $\quad 1$

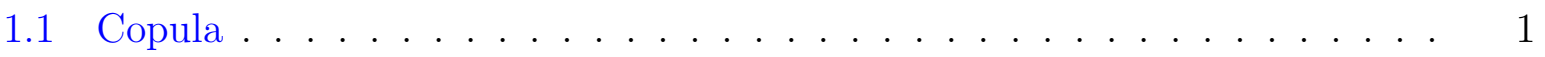

1.2 Risk Measures . . . . . . . . . . . . . . . . . . . . . 2

1.3 Outline of the Thesis . . . . . . . . . . . . . . . . 5

2 Preliminaries $\quad 6$

2.1 MDAs of EVT . . . . . . . . . . . . . . . . . 6

2.1.1 Regular variation and extended regular variation . . . . . . . . 6

2.1.2 Max-domain of Attraction (MDA) . . . . . . . . . . . 8

2.2 Basics of Copula . . . . . . . . . . . . . . . . . . 10

3 Tail Dependence $\quad 14$

3.1 Tail Order Property of Survival Copulas . . . . . . . . . . . . . . . 14

3.2 Uniform Convergence of Survival Copulas . . . . . . . . . . . . . 16 
4 Joint Expected Shortfall (JES) 19

4.1 Asymptotic Analysis . . . . . . . . . . . . . . . . . . . . . . . 19

4.2 Examples . . . . . . . . . . . . . . . . . . . . . . 24

4.3 A Generalization ........................... 27

5 Interplay of Dependence and Heavy Tailedness 30

5.1 A Numerical Example of Gumbel Survival Copula . . . . . . . . . . . . . . 30

5.2 A Numerical Example of Clayton Survival Copula . . . . . . . . . . . . . 31

$\begin{array}{lll}6 & \text { Conclusion } & 37\end{array}$

$\begin{array}{ll}\text { References } & 38\end{array}$

$\begin{array}{ll}\text { APPENDICES } & 42\end{array}$

A Asymptotic Expansions of ES 43

B Numerical Example of Clayton Copula 45

B.1 Figure related calculations . . . . . . . . . . . . . . . . 46

B.2 Table related calculations . . . . . . . . . . . . . . . . 47 


\section{List of Figures}

5.1 From $q=0.95$ to $q=0.999$, the $\log$ of JES is plotted. $X$ and $Y$ are coupled by a Gumbel survival copula with $\kappa=1.5$ and 2. $X$ is Pareto distributed with $\gamma=0.1,0.5$ and $0.9 \ldots \ldots \ldots \ldots \ldots$

5.2 From $q=0.985$ to $q=0.999, \mathbb{E}\left[X \mid X>\zeta F^{\leftarrow}(q), Y>G^{\leftarrow}(q)\right]$ is plotted with $\zeta=0.3,0.7$, and 1. Both MES and ES are plotted for comparison. $X$ and $Y$ follow a Pareto distribution with $\gamma=0.6$ and are coupled by the Clayton survival

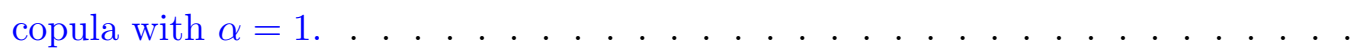




\section{List of Tables}

5.1 The ratio of JES and ES. $X$ and $Y$ follow a Gumbel survival copula. $X$ is Pareto distributed. . . . . . . . . . . . . . . . . . . . . 34

5.2 The ratio of JES and ES. X and Y follow a Clayton survival copula. X is Pareto distributed. . . . . . . . . . . . . . . . . . . 36

5.3 The ratio of JES and MES. X and Y follow a Clayton survival copula. X and Y are Pareto distributed. . . . . . . . . . . . . . . . . . . . . . . . 


\section{Chapter 1}

\section{Introduction}

Much of empirical work has shown that asset returns, exchange rates, operational risks, large insurance claims exhibit heavy tailedness; see e.g. Guillaume et al. (1997), Gabaix et al. (2006), Nešlehová et al. (2006), and Gabaix (2009). Dependence also widely exists among these risks; see Embrechts et al. (2002) for an overview of different dependence measures in risk management. An example of the impact of dependence in finance is the systemic risk observed in the 2008 financial crisis, that is the extreme risks are contagious; see e.g. Acharya et al. (2017). In this thesis, we study the tail dependence through copulas with tail order property and then aim to investigate the effects of heavy tailedness and tail dependence for the extreme risks through a so-called Joint Expected Shortfall risk measure.

\subsection{Copula}

We first study the properties of tail dependence by copulas. Assume that $U$ and $V$ are two uniform random variables with their dependence captured by a copula function $C$, i.e. $(U, V) \sim C$; see the monograph of copula in Nelsen (2006).

Since in this thesis we are interested in the tail dependence, the survival copula $\widehat{C}$ is the focus of the study. In Hua and Joe (2011), the tail order of a copula was showed to 
capture the degree of dependence. That is for $u, v>0$, there exist a tail order $1 \leq \kappa \leq 2$ and a function $\tau(u, v) \geq 0$ such that

$$
\lim _{t \rightarrow 0} \frac{\widehat{C}(u t, v t)}{t^{\kappa} \ell(t)}=\tau(u, v),
$$

where $\ell$ is a slowly varying function. If $\kappa=1$, then $U$ and $V$ are tail dependent; if $1<\kappa \leq 2$, then they are tail independent. When the survival copula function has the tail order property, it is a (multivariate) regularly varying function. In this thesis, we show that if the survival copula $\widehat{C}$ is a regularly varying function (or has the tail order property),

the random vector $\left(V_{1}, V_{2}\right) \sim \widehat{C}$ is multivariate regular variation (MRV) at 0 . For more details of MRV, see e.g. Resnick (2007). This characterization links the tail order property of survival copula with the MRV property so that many well-known results can be applied to copulas, for example, the decomposition of limit measure $\tau$.

Further, we show that the survival copula has a very useful property of uniform convergence, which is parallel to that in the univariate regularly varying functions.

\subsection{Risk Measures}

To study the role of tail dependence in extreme risks, we need to carefully choose the risk measure. The well-known Expected Shortfall (ES) at level $q \in(0,1)$ is defined for a loss random variable $X$ with distribution function $F$ as,

$$
\operatorname{ES}_{q}(X)=\mathbb{E}\left[X \mid X>\operatorname{VaR}_{q}(X)\right]
$$

where $\operatorname{VaR}_{q}(X)=\inf \{x: F(x) \geq q\}$ is the Value-at-Risk of $X$. More strictly, in some literature, ES takes the above form only when the random variable is continuous. In this thesis, we ignore this technical difference and let it be defined for any random variable with finite mean. ES measures the expected loss given that extreme loss of $X$ happens ( $X$ exceeds its VaR limit). ES has many desirable properties, including the coherence; see Artzner et al. (1999). Thus, it has wide applications in finance and insurance, and is 
adopted by the regulators to determine the capital reserve; see McNeil et al. (2015). When it comes to consider a single loss variable $X$ in practice, ES has the disadvantage that it fails to take the dependence into consideration. In the context of systemic risk, Acharya et al. (2017) proposed the following Marginal Expected Shortfall (MES):

$$
\operatorname{MES}=\mathbb{E}\left[X \mid Y>\operatorname{VaR}_{q}(Y)\right]
$$

That is, it exploits the information of extremes in $Y$, instead on the extremes in $X$. MES improves ES by incorporating the information from $Y$ so that the dependence between $X$ and $Y$ is taken into account. In fact, a few variations of MES, by generalizing $Y$ as a function of $X$, have been studied in the literature. Some examples are $Y$ as being a sum of random variables including $X$, or $Y$ being the maximum (minimum) of $n$ random variables including $X$; see e.g. Landsman and Valdez (2003), Cai and Li (2005), Vernic (2006), and Bargès et al. (2009). Besides the explicit calculations of MES under some specific distribution assumptions, the extreme value related studies were all carried out under the tail dependent case, that is $\kappa=1$; see Asimit et al. (2011), Hua and Joe (2014), and Cai et al. (2015). As pointed out in Hua and Joe (2014), the study of MES for the tail independent case $1<\kappa \leq 2$ is technically much more involved. This can be intuitively explained when the dependence between $X$ and $Y$ becomes weaker but not as weak as the independent case, the information provided by $Y$ does not fully reflect the behaviour of $X$. In other words, when $Y$ is in its tail, $X$ is not necessarily in its tail. Thus, when $X$ and $Y$ are tail independent, MES does not necessarily measure the extreme risk of $X$.

To better incorporate the tail independent case in the study of extreme risks, we argue in this thesis that the following newly proposed risk measure can be a better measure of tail risk:

$$
\mathrm{JES}=\mathbb{E}\left[X \mid X>\operatorname{VaR}_{q}(X), Y>\operatorname{VaR}_{q}(Y)\right],
$$

where the joint probability distribution of $(X, Y)$ is a measure on $\mathbb{R}^{2}$. We denote the above risk measure as the Joint Expected Shortfall (JES). Note that JES combines both ES and MES in the sense that the expected loss of $X$ is calculated given the joint information of both $X$ and $Y$ exceeding their corresponding VaR limits. JES can be explained as the 
capital reserve of $X$ when observing both extremes of $X$ and $Y$ occur. In the context of life insurance, JES can be interpreted as when both members in the group survive, what the life expectancy of $X$ is, where $X$ can be modelled with the MDA of Weibull. Also, in the context of property and casualty insurance, JES can be interpreted as when both earthquake and flood (catastrophic events) happen, what is the expected loss caused by one of the catastrophic event. Note that JES can also be written as $\mathbb{E}[X \mid \min (X, Y)>$ $\operatorname{VaR}_{q}(X)$ ], which makes it a special case of MES. The extra information of $X$ makes JES focus on the extreme risk of $X$, which improves MES in the tail independent case. In this thesis, we explore the asymptotic properties of JES when $X$ is from a very general family of distributions, characterized by the max-domains of attraction (MDA). More details of MDA is given in Chapter 2.1.2. One class of distributions in MDA is the regularly varying distributions, which is used to define the heavy tailedness in this thesis. We show that when $X$ belongs to a MDA and $X$ and $Y$ are coupled with a copula with tail order property, as $q$ close to 1 meaning both $X$ and $Y$ are well in their far tails, the JES has a linear relation with $\operatorname{VaR}_{q}(X)$. The linear coefficient is given explicitly and is determined by the dependence and heavy tailedness.

The asymptotic analysis of JES provides an easy and a reliable way to explore the interplay of tail dependence and the heavy tailedness for the extreme risks. While we can always rely on numerical procedures such as the Monte Carlo methods to address these issues, the crude Monte Carlo, however, is notoriously inefficient for rare-event applications, especially for multidimensional case. Through the asymptotic expansions of JES, ES and MES, we find that dependence plays an important role in the measure of extreme risks. In the tail dependent case, the heavy tailedness is a much stronger driving factor than the dependence. By ignoring or solely relying on the dependence structure, measures such as ES and MES may severely underestimate the underlying risk. By jointly conditioning on both $X$ and $Y$, our proposed JES is found to be a more appropriate measure of (extreme) risk. Numerical examples to be presented in subsequent chapter highlight these issues. 


\subsection{Outline of the Thesis}

The rest of thesis is organized as the following. We review the basic theories of EVT and copula in Chapter 2. In Chapter 3, we study the tail dependence through survival copulas. Then, in Chapter 4, we analyze the asymptotic expansions of the proposed JES. The interplay of the dependence and heavy tailedness for the extreme risks is investigated in Chapter 5 through two numerical examples, including the cases of Gumbel and Clayton survival copulas. Chapter 6 concludes the thesis. The asymptotic analysis of ES is presented in Appendix A for completeness, and computations involved in numerical examples are attached in Appendix B. 


\section{Chapter 2}

\section{Preliminaries}

\subsection{MDAs of EVT}

For today's risk management, heavy tailed distributions with negative indexed regularly varying survival function are applied to various models. Due to some mathematical convenience, we focus our analysis on margins belonging to the MDAs. In this chapter, we introduce the basic theory of EVT, and its central idea of MDAs.

First, we introduce the basic concept of regularly varying (RV) functions for EVT. $\mathrm{RV}$ is a key concept in EVT, which plays an important methodological role in modelling tremendous losses for banks and large claims for insurance industry; see Appendix B.1 of de Haan and Ferreira (2006) for more detailed examples of RV functions.

\subsubsection{Regular variation and extended regular variation}

Definition 2.1.1 (RV function) (a) A positive measurable function $l(\cdot)$ is said to be slowly varying at $x_{0}=+0$ or $+\infty$, denoted by $l(\cdot) \in \mathrm{RV}_{0}\left(x_{0}\right)$, if

$$
\lim _{x \rightarrow x_{0}} \frac{l(x t)}{l(x)}=1, t>0
$$


(b) A positive measurable function $f(\cdot)$ is said to be regularly varying at $x_{0}=+0$ or $+\infty$ with index $\alpha \in \mathbb{R}$, denoted by $f(\cdot) \in \mathrm{RV}_{\alpha}\left(x_{0}\right)$, if

$$
\lim _{x \rightarrow x_{0}} \frac{f(x t)}{f(x)}=t^{\alpha}, t>0
$$

Next, we present a very useful inequality for RV functions; refer to Proposition B.1.9 of de Haan and Ferreira (2006).

Lemma 2.1.1 (Potter's bound) Let $f(\cdot) \in \mathrm{RV}_{\alpha}\left(x_{0}\right)$ with $x_{0}=+0$ or $+\infty$ and $\alpha \in \mathbb{R}$. It holds for arbitrary $0<\varepsilon<1$ and all $x, y$ sufficiently close to $x_{0}$ that

$$
(1-\varepsilon)\left(\left(\frac{y}{x}\right)^{\alpha+\varepsilon} \wedge\left(\frac{y}{x}\right)^{\alpha-\varepsilon}\right) \leq \frac{f(y)}{f(x)} \leq(1+\varepsilon)\left(\left(\frac{y}{x}\right)^{\alpha+\varepsilon} \vee\left(\frac{y}{x}\right)^{\alpha-\varepsilon}\right)
$$

where $\wedge$ is the minimum operator, and $\vee$ is the maximum operator. Before describing MDA, it is useful to first introduce the concept of extended regular variation (ERV). It serves an important role in the characterization of MDAs.

Definition 2.1.2 (ERV function) A positive measurable function $f(\cdot)$ is said to be extended regularly varying at $\infty$ with index $\gamma \in \mathbb{R}$, denoted by $f(\cdot) \in \mathrm{ERV}_{\gamma}$, if there is an auxiliary function $a(\cdot)>0$ such that, for all $s>0$,

$$
\lim _{t \rightarrow \infty} \frac{f(s t)-f(t)}{a(t)}=\frac{s^{\gamma}-1}{\gamma}
$$

where the right-hand-side is interpreted as $\log s$ when $\gamma=0$. The auxiliary function $a(\cdot)$ is often chosen to be

$$
a(t)= \begin{cases}\gamma f(t), & \gamma>0 \\ f(t)-t^{-1} \int_{0}^{t} f(u) \mathrm{d} u, & \gamma=0 \\ -\gamma(f(\infty)-f(t)), & \gamma<0\end{cases}
$$

Note that, for $\gamma=0$, as $t \rightarrow \infty$, we have $a(t)=o(f(t))$ provided $f(\infty)=\infty$ while $a(t)=o(f(\infty)-f(t))$ provided $f(\infty)<\infty$. 
In particular, if $\gamma>0$, ERV is the well known regular variation $(\mathrm{RV})$. That is, $f(\cdot)$ is regularly varying at $t_{0}=\infty$ or $0+$, denoted by $f(\cdot) \in \mathrm{RV}_{\gamma}\left(t_{0}\right)$, if

$$
\lim _{t \rightarrow t_{0}} \frac{f(s t)}{f(t)}=s^{\gamma}, \quad s>0
$$

The class $\mathrm{RV}_{0}\left(t_{0}\right)$ consists of functions slowly varying at $t_{0}$.

The following theorem specifies the upper and lower bounds for ERV functions, which is similar to the Potter's bound for RV functions.

Theorem 2.1.1 If $f(\cdot) \in \mathrm{ERV}_{\gamma}$, then for any $\epsilon, \delta>0$, there exists some $x_{0}=x_{0}(\epsilon, \delta)$ such that for all $x, x y>x_{0}$,

$$
\left|\frac{f(x y)-f(x)}{a_{0}(x)}-\frac{y^{\gamma}-1}{\gamma}\right| \leq \epsilon y^{\gamma} \max \left\{y^{\delta}, y^{-\delta}\right\},
$$

The interested readers may refer to Appendix B of de Haan and Ferreira (2006) for more discussions on ERV.

\subsubsection{Max-domain of Attraction (MDA)}

Now we are ready to introduce the MDAs of EVT.

Definition 2.1.3 (MDA) Let $X_{1}, X_{2}, \ldots X_{n}$ be a sequence of i.i.d random variables with common distribution function $F$. Then the block maxima is defined as $M_{n}=\max \left\{X_{1}, \ldots, X_{n}\right\}$. Suppose there exists normalizing sequences of real numbers $\left(c_{n}\right)>0$ and $\left(d_{n}\right) \in \mathbb{R}$ such that $\left(M_{n}-d_{n}\right) / c_{n}$ converges in distribution, i.e.

$$
\operatorname{Pr}\left(\left(M_{n}-d_{n}\right) / c_{n}<x\right) \rightarrow H(x), n \rightarrow \infty
$$

for some non-degenerate $d f H$ (not a unit jump). Then $F$ is said to belong to the maximum domain of attraction of $H$, and it is denoted by $F \in M D A(H)$. 
With a focus on the limiting behaviour of the centred and normalized sample maxima, the Fisher-Tippett theorem of EVT is analogous to the central limit theorem.

Theorem 2.1.2 (Fisher-Tippett theorem) Let $X_{1}, X_{2}, \ldots X_{n}$ be a sequence of i.i.d random variables with common distribution function $F$. If there exists normalizing constants $\left(c_{n}\right)>$ 0 and $\left(d_{n}\right)$ such that $\left(M_{n}-d_{n}\right) / c_{n}$ converges in distribution, i.e.

$$
\operatorname{Pr}\left(\left(M_{n}-d_{n}\right) / c_{n}<x\right) \rightarrow H(x), n \rightarrow \infty
$$

for some non-degenerate $d f H$ (not a unit jump), then $H$ is of the type of one of the following three extreme value distributions:

(a) Fréchet $(\gamma>0)$ :

$$
\Phi_{\gamma}(x)= \begin{cases}0, & x \leq 0, \\ \exp \left\{-x^{-\gamma}\right\}, & x>0\end{cases}
$$

(b) Gumbel $(\gamma=0)$ :

$$
\Lambda_{\gamma}(x)=\exp \left\{-x^{-\gamma}\right\}, x \in \mathbb{R}
$$

(c) Weibull $(\gamma>0)$ :

$$
\Psi_{\gamma}(x)= \begin{cases}\exp \left\{-|x|^{\gamma}\right\}, & x \leq 0, \\ 1, & x>0\end{cases}
$$

Note that theorem is critical for us to classify heavy-tailed distributions into one of the three categories.

In the Fréchet class, the distributions are heavy tailed, more specifically, they have regularly varying tails. Typical examples include Pareto, student- $t$, and Cauchy distributions. Thus, distributions in this category are usually common models for large fluctuations of prices and large losses in finance and insurance. The Gumbel class covers a wide range of 
distributions, including exponential, Gamma, Normal, and Lognormal distributions. Distributions in the Weibull class are bounded and are regularly varying at the upper end point, which includes Beta and Uniform distributions.

The MDA of generalized extreme value distribution is related to ERV through the tail quantile function

$$
U(t)=\left(\frac{1}{\bar{F}}\right)^{\leftarrow}(t)=F^{\leftarrow}\left(1-\frac{1}{t}\right), \quad t>1 .
$$

We have $F \in \operatorname{MDA}\left(G_{\gamma}\right)$ if and only if $U(\cdot) \in \operatorname{ERV}_{\gamma}$ with the auxiliary function $a(\cdot)$ given in (2.1.1) in terms of $U(\cdot)$, where

$$
G_{\gamma}= \begin{cases}\Phi_{\frac{1}{\gamma}}, & \gamma>0 \\ \Psi_{-\frac{1}{\gamma}}, & \gamma<0 \\ \Lambda, & \gamma=0\end{cases}
$$

see Theorem 1.1.6 of de Haan and Ferreira (2006) for details.

\subsection{Basics of Copula}

In this section, we make a brief review on basic theory on copulas.; for a complete overview see Nelsen (2006).

We introduce the theory of bivariate copulas, while it can be naturally extended to multivariate case.

Definition 2.2.1 (Copula) A two-dimensional copula is a function $C:[0,1]^{2} \rightarrow[0,1]$ satisfying the following properties:

(a) Boundary conditions: $C(0, u)=C(u, 0)=0$, and $C(1, u)=C(u, 1)=u$ for all $u \in$ $[0,1]$. 
(b) 2-increasingness: For every $0 \leq u_{1}<u_{2} \leq 1$ and $0 \leq v_{1}<v_{2} \leq 1$,

$$
C\left(u_{2}, v_{2}\right)-C\left(u_{1}, v_{2}\right)-C\left(u_{2}, v_{1}\right)+C\left(u_{1}, v_{1}\right) \geq 0 .
$$

A $d$-dimensional copula for $d \geq 3$ can be defined in a similar way; see Nelsen (2006) in details.

By Definition 2.2.1, one can see that copula is a 2-dimensional cumulative distribution function whose marginal distributions are uniform on $[0,1]$.

Since our analysis is focused on the tail risks, the idea of survival copula will bring us more convenience for the asymptotic analysis. Here, the fundamental idea of survival copula and its relation with copula are reviewed.

Definition 2.2.2 (Survival copula) The survival copula associated with $C(u, v)$ is defined as

$$
\hat{C}(u, v)=\operatorname{Pr}(1-U \leq u, 1-V \leq v)
$$

and

$$
\hat{C}(u, v)=u+v-1+C(1-u, 1-v) .
$$

Note that $\hat{C}(\bar{F}(x), \bar{G}(y))=\operatorname{Pr}(X>x, Y>y)$, where $\bar{F}$ is the survival function of $X$, and $\bar{G}$ is the survival function of Y. $\hat{C}$ is also a copula function.

Any copula is bounded from above and below by the following functions with respect to the concordance order.; see Theorem 2.2.3 of Nelsen (2006) for detailed reference.

Theorem 2.2.1 (Fréchet-Höffding bounds) Let $W(\boldsymbol{u})=\max \left\{\sum_{j=1}^{d} u_{j}-d+1,0\right\}$ and $M(\boldsymbol{u})=$ $\min _{1 \leq j \leq d}\left\{u_{j}\right\}$

(a) For any d-dimensional copula $C$,

$$
W(\boldsymbol{u}) \leq C(\boldsymbol{u}) \leq M(\boldsymbol{u}), \boldsymbol{u} \in[0,1]^{d} .
$$


(b) $W$ is a copula if and only if $d=2$.

(c) $M$ is a copula for all $d \geq 2$.

$W$ and $M$ are regarded as the widest bounds of copulas. $W$ is the countermonoticity copula, and $M$ is the comonotonicity copula. They are the fundamental copulas.

Another important fundamental copula is the independent copula. The independence copula is defined as: $\Pi(\boldsymbol{u})=\prod_{j=1}^{d} u_{j}, \boldsymbol{u} \in[0,1]^{d}$.

The following theorem is a building block of the copula theory. It illustrates how the joint distribution can be represented by copula and marginal distributions, and how the copula can be defined by the joint distribution and marginal distributions; see Theorem 2.3.3 of Nelsen (2006) for more details.

Theorem 2.2.2 (Sklar's Theorem) (a) For any distribution function F with margins $F_{1}, \ldots, F_{d}$, there exists a copula $C$ such that

$$
F\left(x_{1}, \ldots, x_{d}\right)=C\left(F_{1}\left(x_{1}\right), \ldots, F_{d}\left(x_{d}\right)\right), x \in \mathbb{R}^{d}
$$

where $C$ is uniquely defined on $\prod_{j=1}^{d}$ ran $F_{j}$ and given by

$$
C\left(u_{1}, \ldots, u_{d}\right)=F\left(F_{1}^{\leftarrow}\left(u_{1}\right), \ldots, F_{d}^{\leftarrow}\left(u_{d}\right)\right), u \in \prod_{j=1}^{d} \operatorname{ran} F_{j}
$$

where ran $F_{j}=\left\{F_{j}(x): x \in R\right\}$ denotes the range of $F_{j}$.

(b) Conversely, given any copula $C$ and univariate distribution functions, $F$ defined by (2.2.2) is a distribution function with margins $F_{1}, \ldots, F_{d}$.

Last but not least, we introduce the structure of an extensively studied family of Archimedean copulas, which are helpful for modelling portfolio credit risks. We will include 
numerical examples of Archimedean copulas in chapter 4, including Clayton and Gumbel copulas.

Definition 2.2.3 (Archimedean copula) Archimedean copulas are copulas of the form $C(\boldsymbol{u})=$ $\psi\left(\psi^{-1}\left(u_{1}\right)+\ldots+\psi^{-1}\left(u_{d}\right)\right)$, where $\psi$ is the Archimedean generator such that:

(a) $\psi:[0, \infty) \rightarrow[0,1]$

(b) $\psi$ is decreasing on $[0, \inf \{t: \psi(t)=0\}]$

(c) $\psi(0)=1, \psi(\infty)=\lim _{t \rightarrow \infty} \psi(t)=0$

For instance, the generator of Clayton copula is $\psi(t)=(1+t)^{-\frac{1}{\theta}}, t \in[0, \infty), \theta \in(0, \infty)$. As $\theta \downarrow 0, C \rightarrow \Pi$, and for $\theta \uparrow \infty, C \rightarrow M$. On the other hand, the generator function of Gumbel copula is $\psi(t)=\exp \left(-t^{1 / \theta}\right), t \in[0, \infty), \theta \in[1, \infty)$. For $\theta=1, C=\Pi$, and as $\theta \uparrow \infty, C \rightarrow M$. 


\section{Chapter 3}

\section{Tail Dependence}

The focus of this chapter is to study the tail properties of survival copulas. We first define the survival copulas with tail order property through regular variation. Starting from this definition, the random vector $\left(V_{1}, V_{2}\right) \sim \widehat{C}$ is showed to be multivariate regular variation (MRV) at 0. A uniform convergence property is investigated for these copulas, which is useful in our subsequent studies of extreme risks.

\subsection{Tail Order Property of Survival Copulas}

We first present the regular variation version of the tail order property for survival copulas.

Assumption 3.1.1 There exists a non-degenerate and continuous tail function $\tau\left(v_{1}, v_{2}\right) \not \equiv$ 0 such that for any $v_{1}, v_{2} \geq 0$,

$$
\lim _{t \downarrow 0} \frac{\widehat{C}\left(v_{1} t, v_{2} t\right)}{\widehat{C}(t, t)}=\tau\left(v_{1}, v_{2}\right)
$$

Note that, since $\widehat{C}\left(0, v_{2}\right)=\widehat{C}\left(v_{1}, 0\right)=\widehat{C}(0,0)=0$, it is naturally to have $\tau\left(0, v_{2}\right)=$ $\tau\left(v_{1}, 0\right)=\tau(0,0)=0$. Thus, (3.1.1) is well defined for any $v_{1}, v_{2} \geq 0$. Many widely 
used copulas, including the extreme value survival copulas and Archimedean survival copulas with regularly varying generator, satisfy Assumption 3.1.1; more details are given in Chapter 4.2 .

Assumption 3.1.1 indeed assumes that $\widehat{C}(\cdot, \cdot)$ is multivariate regularly varying (MRV) on $[0, \infty)^{2}$. Then there is some $\kappa$ such that

$$
\tau\left(v_{1} t, v_{2} t\right)=t^{\kappa} \tau\left(v_{1}, v_{2}\right)
$$

Here $\kappa$ is the so-called tail order and takes values in [1,2]; see e.g. Hua and Joe (2011). In fact, (3.1.1) can be formulated using the tail order as the follows: there exists a slowly varying function $\ell(t)$ (the definition of slowly varying can be found in Section 2.1.2), and a non-degenerate tail function $b\left(v_{1}, v_{2}\right) \not \equiv 0$ such that, for any $v_{1}, v_{2} \geq 0$,

$$
\lim _{t \downarrow 0} \frac{\widehat{C}\left(v_{1} t, v_{2} t\right)}{t^{\kappa} \ell(t)}=b\left(v_{1}, v_{2}\right) .
$$

It is obvious that $\tau\left(v_{1}, v_{2}\right)=b\left(v_{1}, v_{2}\right) / b(1,1)$.

The MRV of $\widehat{C}(\cdot, \cdot)$ (i.e. Assumption 3.1.1) should not be confused with the usual one that is assumed for random variables with regularly varying tails at infinity. Here, $\widehat{C}(\cdot, \cdot)$ has marginals of uniform distributions, which are regularly varying at 0 . Thus, it is important to note that the MRV property for $\widehat{C}(\cdot, \cdot)$ is defined on $[0, \infty)^{2}$. The infinity is excluded due to that the tail events in the study of $\widehat{C}(\cdot, \cdot)$ are around $(0,0)$.

Parallel to the discussion of MRV in Chapter 6 of Resnick (2007), we construct a similar vague convergence for $\widehat{C}(\cdot, \cdot)$ in Proposition 3.1.1. One may refer Resnick (2007) for the technical terms such as vague convergence and Radon measure. Both Propositions 3.1.1 and 3.2.1 are important properties of copulas satisfying Assumption 3.1.1 and help us to better understand them.

Proposition 3.1.1 Let $\mathbf{V}=\left(V_{1}, V_{2}\right)$ be a random vector of uniform distributions. Suppose V follows the copula $\widehat{C}(\cdot, \cdot)$ which satisfies Assumption 3.1.1. Then there exist a function 
$b(t) \rightarrow 0$ and a Radon measure $\tau$ on $[0, \infty)^{2}$, such that in $\mathcal{B}\left([0, \infty)^{2}\right)$,

$$
\frac{1}{t} \operatorname{Pr}\left(\frac{\mathbf{V}}{b(t)} \in \cdot\right) \stackrel{\mathrm{v}}{\rightarrow} \tau, \quad t \rightarrow 0,
$$

where $\stackrel{\mathrm{v}}{\rightarrow}$ denotes the vague convergence.

Proof. From Assumption 3.1.1, $\widehat{C}(\cdot, \cdot)$ is a multivariate regularly varying function on $[0, \infty)^{2}$. It implies that $\widehat{C}(t, t)$ is a regularly varying function of $t$ : there exists some $\kappa>0$ and a slowly varying function $\ell(t)$ such that when $t$ is close to 0

$$
\widehat{C}(t, t)=t^{\kappa} \ell(t)
$$

Define $b(t)$ such that

$$
\lim _{t \rightarrow 0} \frac{\widehat{C}(b(t), b(t))}{t}=1 .
$$

Thus $b(t) \in \mathrm{RV}_{1 / \kappa}(0)$. Then replacing $t$ by $b(t)$ in (3.1.1) yields the weak convergence for $\mathbf{v}=\left(v_{1}, v_{2}\right)^{T} \in[0, \infty)^{2}$

$$
\lim _{t \downarrow 0} \frac{1}{t} \widehat{C}(u b(t), v b(t))=\lim _{t \downarrow 0} \frac{1}{t} \operatorname{Pr}\left(\frac{\mathbf{V}}{b(t)} \in[\mathbf{0}, \mathbf{v}]\right)=\tau\left(v_{1}, v_{2}\right) .
$$

Denote the measure $\frac{1}{n} \operatorname{Pr}\left(\frac{\mathbf{V}}{b(n)} \in \cdot\right)$ by $\tau_{n}$. Then the above weak convergence is indeed

$$
\tau_{n}([\mathbf{0}, \mathbf{v}]) \rightarrow \tau([\mathbf{0}, \mathbf{v}]), \quad \text { as } n \rightarrow \infty .
$$

Similar to the proof of Lemma 6.1 in Resnick (2007), we can show that $\tau_{n} \stackrel{\mathrm{v}}{\rightarrow} \tau$.

\subsection{Uniform Convergence of Survival Copulas}

Another property for $\widehat{C}(\cdot, \cdot)$ is the uniform convergence on a compact set. This has been shown under the special case that $\kappa=1$ in Schmidt and Stadtmüller (2006). Here we 
extend it to the most general form for all dependence with $1 \leq \kappa \leq 2$. This result is useful for the proofs in Chapter 4.

Proposition 3.2.1 Under Assumption 3.1.1, we have that for any compact set $B \in$ $[0, \infty)^{2}$

$$
\lim _{t \downarrow 0} \sup _{u, v \in B}\left|\frac{\widehat{C}(u t, v t)}{\widehat{C}(t, t)}-\tau(u, v)\right|=0 .
$$

Proof. Fix a compact set $B$. We prove the proposition by contradiction. Suppose the assertion in the proposition does not hold. Without loss of generality, there exist some $\varepsilon_{0}>0$ and sequences $\left\{t_{n}\right\},\left\{u_{n}\right\}$ and $\left\{v_{n}\right\}$ such that

$$
\frac{\widehat{C}\left(u_{n} t_{n}, v_{n} t_{n}\right)}{\widehat{C}\left(t_{n}, t_{n}\right)}-\tau\left(u_{n}, v_{n}\right)>\varepsilon_{0},
$$

where $t_{n}$ converges to 0 as $n \rightarrow \infty$. Since the sequences $\left\{u_{n}\right\}$ and $\left\{v_{n}\right\}$ are bounded, by the Bolzano-Weierstrass Theorem there exist convergent subsequences $\left\{u_{n_{k}}\right\}$ and $\left\{v_{n_{k}}\right\}$ with $u_{n_{k}} \rightarrow u_{0}, v_{n_{k}} \rightarrow v_{0}$ as $n_{k} \rightarrow \infty$. We choose $0<\varepsilon<\varepsilon_{0} / 2$ and $\delta>0$ such that when $n_{k}$ is large $u_{0}-\delta<u_{n_{k}}<u_{0}+\delta, v_{0}-\delta<v_{n_{k}}<v_{0}+\delta$ (if $u_{0}=0$ or $v_{0}=0$, then we replace the previous inequality by $0<u_{n_{k}}<\delta$ or $0<v_{n_{k}}<\delta$ ), and

$$
\tau\left(u_{0}+\delta, v_{0}+\delta\right)-\tau\left(u_{0}-\delta, v_{0}-\delta\right)<\varepsilon
$$

Note that $\widehat{C}(u, v)$ and $\tau(u, v)$ are both non-decreasing in $u$ and $v$. By using (3.2.1) together with the monotonicity of $\widehat{C}$ and $b$, we have

$$
\begin{aligned}
\varepsilon_{0} & <\frac{\widehat{C}\left(u_{n_{k}} t_{n_{k}}, v_{n_{k}} t_{n_{k}}\right)}{\widehat{C}\left(t_{n_{k}}, t_{n_{k}}\right)}-\tau\left(u_{n_{k}}, v_{n_{k}}\right) \\
& \leq \frac{\widehat{C}\left(\left(u_{0}+\delta\right) t_{n_{k}},\left(v_{0}+\delta\right) t_{n_{k}}\right)}{\widehat{C}\left(t_{n_{k}}, t_{n_{k}}\right)}-\tau\left(u_{0}-\delta, v_{0}-\delta\right) \\
& \leq \frac{\widehat{C}\left(\left(u_{0}+\delta\right) t_{n_{k}},\left(v_{0}+\delta\right) t_{n_{k}}\right)}{\widehat{C}\left(t_{n_{k}}, t_{n_{k}}\right)}-\tau\left(u_{0}+\delta, v_{0}+\delta\right)+\varepsilon
\end{aligned}
$$


Thus,

$$
\lim _{n_{k} \rightarrow \infty}\left(\frac{\widehat{C}\left(\left(u_{0}+\delta\right) t_{n_{k}},\left(v_{0}+\delta\right) t_{n_{k}}\right)}{\widehat{C}\left(t_{n_{k}}, t_{n_{k}}\right)}-\tau\left(u_{0}+\delta, v_{0}+\delta\right)\right)>\frac{\varepsilon_{0}}{2},
$$

which contradicts to Assumption 3.1.1. 


\section{Chapter 4}

\section{Joint Expected Shortfall (JES)}

In this chapter, the asymptotic expansions for JES are derived by applying MDAs to model margins. Some examples are presented to illustrate our results. Finally, a generalization of JES is introduced.

For two positive functions $f(\cdot)$ and $g(\cdot)$, we write $f(\cdot) \sim g(\cdot)$ if the ratio of left-hand side (LHS) and right-hand side (RHS) converges to 1 ; that is, $\lim f(\cdot) / g(\cdot)=1$.

\subsection{Asymptotic Analysis}

In this section we focus on the asymptotic analysis of the JES. Besides Assumption 3.1.1 proposed in Chapter 3, we further need to impose some regularity to the limit function $\tau(\cdot, \cdot)$.

Assumption 4.1.1 Assume there exists $\beta>0, \xi>0$ and a positive bounded function $h \in \mathrm{RV}_{0}(0+)$ such that $\tau(x, 1)=x^{\beta} h(x)$ for $0 \leq x \leq \xi$.

Assumption 4.1.1 assumes that $\tau(\cdot, 1)$ is regularly varying at 0 with index $\beta$. Commonly used copulas satisfy this assumption; details are given in Chapter 4.2. With the bounded 
function $h, \tau(x, 1)$ is then bounded by a power function. In the next lemma we show that the index $\beta$ helps to construct a uniform bound on the survival copula $\widehat{C}$, which parallels to the Potter's bound in the univariate case. This plays a key role in the convergence of $\widehat{C}$ as can be seen in Lemma 4.1.2.

Lemma 4.1.1 Assume that $\widehat{C}$ is a survival copula satisfying Assumptions 3.1.1 and 4.1.1. Then there exist $c>0$ and $t_{0}>0$ such that for all $t>t_{0}$ and $x \in[0, \xi]$,

$$
\frac{\widehat{C}(x / t, 1 / t)}{\widehat{C}(1 / t, 1 / t)} \leq c x^{\beta} .
$$

Proof. Let

$$
f_{n}(x)=\frac{\widehat{C}\left(\frac{x}{n}, \frac{1}{n}\right)}{\widehat{C}\left(\frac{1}{n}, \frac{1}{n}\right)} .
$$

Since $f_{n}(0)=0$, it suffices to show that: there exists $N>0$ and $c>0$ such that for $n>N$ and all $x \in(0, \xi], f_{n}(x) \leq c x^{\beta}$. Suppose not, then for each $c>0$ and every $N$, there exist $n>N$ and $y>0$ such that $f_{n}(y)>c y^{\beta}$. On the other hand, by Proposition 3.2.1, for any $\varepsilon>0$, there is $M>0$ such that for $n>M$ and $x \in[0, \xi], f_{n}(x) \leq \tau(x, 1)+\varepsilon$. Further by Assumption 4.1.1 there is $c_{0}>0$ such that $f_{n}(x) \leq c_{0} x^{\beta}+\varepsilon$. Then for each $c_{k}>c_{0}$, $k=1,2,3, \ldots$, there exist $n_{k}>M$ and $y_{k}>0$ such that

$$
c_{k} y_{k}^{\beta}<f_{n_{k}}\left(y_{k}\right)<c_{0} y_{k}^{\beta}+\varepsilon
$$

If $y_{k} \geq\left(\frac{\varepsilon}{c_{k}-c_{0}}\right)^{1 / \beta}$, or equivalently, $c_{k} y_{k}^{\beta} \geq c_{0} y_{k}^{\beta}+\varepsilon$, then it contradicts to (4.1.1). If $y_{k}<\left(\frac{\varepsilon}{c_{k}-c_{0}}\right)^{1 / \beta}$, then as $c_{k}$ increasing, $y_{k}$ converges to 0 . That is, for any $\varepsilon$, the only possible value of $y$ for (4.1.1) to hold for all $c_{k}>0$ is 0 . This contradicts to that (4.1.1) holds for some $y>0$.

Next we show a key lemma in the proof of the main result. It is constructed for all three MDAs in a unified way. 
Lemma 4.1.2 Assume that $\widehat{C}$ is a survival copula satisfying Assumptions 3.1.1 and 4.1.1. Suppose that a tail quantile function $U \in \operatorname{ERV}_{\gamma}$ with $\gamma<1 \wedge \beta$ and auxiliary function $a(\cdot)$. Then we have as $q \uparrow 1$

$$
\lim _{t \rightarrow \infty} \frac{1}{\widehat{C}(1 / t, 1 / t)} \int_{0}^{1} \frac{U(t / x)-U(t)}{a(t)} \mathrm{d} \widehat{C}\left(\frac{x}{t}, \frac{1}{t}\right)=\int_{0}^{1} \frac{x^{-\gamma}-1}{\gamma} \mathrm{d} \tau(x, 1) .
$$

Proof. By Theorem B.2.18 of de Haan and Ferreira (2006), for $\varepsilon, \delta>0$ there exists $t_{1}>0$ such that for any $t>t_{1}$ and $0<x \leq 1$

$$
\left|\frac{U(t / x)-U(t)}{a(t)}-\frac{x^{-\gamma}-1}{\gamma}\right| \leq \varepsilon x^{-\gamma-\delta}
$$

Then

$$
\frac{1}{\widehat{C}(1 / t, 1 / t)} \int_{0}^{1} \frac{U(t / x)-U(t)}{a(t)} \mathrm{d} \widehat{C}\left(\frac{x}{t}, \frac{1}{t}\right) \leq \int_{0}^{1}\left(\frac{x^{-\gamma}-1}{\gamma}+\varepsilon x^{-\gamma-\delta}\right) \frac{\mathrm{d} \widehat{C}(x / t, 1 / t)}{\widehat{C}(1 / t, 1 / t)} .
$$

Note that by Assumption 3.1.1, for any $x \geq 0$,

$$
\lim _{t \rightarrow \infty} \frac{\widehat{C}(x / t, 1 / t)}{\widehat{C}(1 / t, 1 / t)}=\tau(x, 1)
$$

It suffices to analyse if the following holds:

$$
\lim _{t \rightarrow \infty} \int_{0}^{1} x^{-\gamma-\delta} \frac{\mathrm{d} \widehat{C}(x / t, 1 / t)}{\widehat{C}(1 / t, 1 / t)}=1-\lim _{t \rightarrow \infty} \int_{0}^{1} \frac{\widehat{C}(x / t, 1 / t)}{\widehat{C}(1 / t, 1 / t)} \mathrm{d} x^{-\gamma-\delta}=1-\int_{0}^{1} \tau(x, 1) \mathrm{d} x^{-\gamma-\delta}
$$

that is, the limit and the integral are interchangeable in the second step above.

First note that by Proposition 3.2.1 the convergence in (4.1.3) is uniform for $x$ in any compact set. Then for any $0<\xi<1$,

$$
\lim _{t \rightarrow \infty} \int_{\xi}^{1} \frac{\widehat{C}(x / t, 1 / t)}{\widehat{C}(1 / t, 1 / t)} \mathrm{d} x^{-\gamma-\delta}=\lim _{t \rightarrow \infty} \int_{\xi}^{1} \tau(x, 1) \mathrm{d} x^{-\gamma-\delta}
$$


Now for $x \in[0, \xi]$, since $\gamma<1 \wedge \beta$ and $\delta$ can be chosen so that $\gamma+\delta<1 \wedge \beta$, by Lemma 4.1.1 there exists $c>0$ such that

$$
\int_{0}^{\xi} \frac{\widehat{C}(x / t, 1 / t)}{\widehat{C}(1 / t, 1 / t)} \mathrm{d} x^{-\gamma-\delta} \leq c \int_{0}^{\xi} x^{\beta} \mathrm{d} x^{-\gamma-\delta} \leq \frac{c}{\beta-\gamma-\delta}<\infty .
$$

Thus, by the dominated convergence theorem,

$$
\lim _{t \rightarrow \infty} \frac{1}{\widehat{C}(1 / t, 1 / t)} \int_{0}^{1} x^{-\gamma-\delta} \mathrm{d} \widehat{C}\left(\frac{x}{t}, \frac{1}{t}\right)=\int_{0}^{1} x^{-\gamma-\delta} \mathrm{d} \tau(x, 1)
$$

Further we have

$$
\lim _{t \rightarrow \infty} \frac{1}{\widehat{C}(1 / t, 1 / t)} \int_{0}^{1} \frac{U(t / x)-U(t)}{a(t)} \mathrm{d} \widehat{C}\left(\frac{x}{t}, \frac{1}{t}\right) \leq \int_{0}^{1}\left(\frac{x^{-\gamma}-1}{\gamma}+\varepsilon x^{-\gamma-\delta}\right) \mathrm{d} \tau(x, 1) .
$$

The other side of the inequality can be constructed similarly. By the arbitrariness of $\varepsilon$ and $\delta$, the desired result follows.

We now present the asymptotic expansions for the JES.

Theorem 4.1.1 Suppose that $X$ follows a distribution function $F$ with a tail quantile function $U \in \operatorname{ERV}_{\gamma}$ with $\gamma<1 \wedge \beta$ and auxiliary function $a(\cdot)$, and $Y$ follows a distribution function $G$. Let $\widehat{C}$ be the survival copula of $(X, Y)$ satisfying Assumptions 3.1.1 and 4.1.1. Then we have as $q \uparrow 1$,

(i) Fréchet case $(\gamma>0)$ :

$$
\mathbb{E}\left[X \mid X>F^{\leftarrow}(q), Y>G^{\leftarrow}(q)\right] \sim F^{\leftarrow}(q)\left(1+\int_{1}^{\infty} \tau\left(x^{-1 / \gamma}, 1\right) \mathrm{d} x\right)
$$

(ii) Gumbel case $(\gamma=0)$ : if $\hat{x}=\infty$, then

$$
\mathbb{E}\left[X \mid X>F^{\leftarrow}(q), Y>G^{\leftarrow}(q)\right] \sim F^{\leftarrow}(q),
$$


if $\hat{x}<\infty$, then

$$
\hat{x}-\mathbb{E}\left[X \mid X>F^{\leftarrow}(q), Y>G^{\leftarrow}(q)\right] \sim \hat{x}-F^{\leftarrow}(q)
$$

(iii) Weibull case $(\gamma<0)$ :

$$
\hat{x}-\mathbb{E}\left[X \mid X>F^{\leftarrow}(q), Y>G^{\leftarrow}(q)\right] \sim\left(\hat{x}-F^{\leftarrow}(q)\right)\left(1-\int_{0}^{1} \tau\left(x^{-1 / \gamma}, 1\right) \mathrm{d} x\right) .
$$

Proof. Let $V$ and $W$ be two uniformly distributed random variables on $[0,1]$. Then, $X \stackrel{d}{=} F^{\leftarrow}(1-V)=U\left(\frac{1}{V}\right)$, and $Y \stackrel{d}{=} G^{\leftarrow}(1-W)$. By letting $t=1 /(1-q)$, we have

$$
\begin{aligned}
\mathbb{E}\left[X \mid X>F^{\leftarrow}(q), Y>G^{\leftarrow}(q)\right] & =\mathbb{E}\left[U\left(\frac{1}{V}\right) \mid V<\frac{1}{t}, W<\frac{1}{t}\right] \\
& =\frac{1}{\widehat{C}(1 / t, 1 / t)} \int_{0}^{1 / t} U\left(\frac{1}{v}\right) \mathrm{d} \widehat{C}(v, 1 / t) \\
& =\frac{1}{\widehat{C}(1 / t, 1 / t)} \int_{0}^{1} U\left(\frac{t}{x}\right) \mathrm{d} \widehat{C}\left(\frac{x}{t}, \frac{1}{t}\right) \\
& =\frac{a(t)}{\widehat{C}(1 / t, 1 / t)} \int_{0}^{1} \frac{U(t / x)-U(t)}{a(t)} \mathrm{d} \widehat{C}\left(\frac{x}{t}, \frac{1}{t}\right)+U(t) .
\end{aligned}
$$

Next, we consider the three classes separately.

(i) For $\gamma>0, \hat{x}=\infty$, and the auxiliary function $a(t)=\gamma U(t)$. Note that $\tau(0,1)=0$ and $U(t)=F^{\leftarrow}(q)$. Since $\hat{x}=\infty$, by Lemma 4.1 .2 we have

$$
\lim _{q \rightarrow 1} \frac{\mathbb{E}\left[X \mid X>F^{\leftarrow}(q), Y>G^{\leftarrow}(q)\right]}{F^{\leftarrow}(q)}=\gamma \int_{0}^{1} \frac{x^{-\gamma}-1}{\gamma} \mathrm{d} \tau(x, 1)+1=1+\int_{1}^{\infty} \tau\left(x^{-1 / \gamma}, 1\right) \mathrm{d} x .
$$

(ii) For $\gamma=0$, two cases arise. If $\hat{x}<\infty$, then $a(t)=o(\hat{x}-U(t))$, and $\mathbb{E}[X \mid X>$ $\left.F^{\leftarrow}(q), Y>F^{\leftarrow}(q)\right]$ and $F^{\leftarrow}(q)$ converge to $\hat{x}$. It follows from (4.1.4) and Lemma 4.1.2 that

$$
\frac{\hat{x}-\mathbb{E}\left[X \mid X>F^{\leftarrow}(q), Y>F^{\leftarrow}(q)\right]}{\hat{x}-U(t)}=1-o(\hat{x}-U(t)) .
$$


If $\hat{x}=\infty$, then $a(t)=o(U(t))$. From (4.1.4) and Lemma 4.1.2, we obtain

$$
\frac{\mathbb{E}\left[X \mid X>F^{\leftarrow}(q), Y>F^{\leftarrow}(q)\right]}{U(t)}=1+o(U(t)) .
$$

In both cases, the desired results follow.

(iii) For $\gamma<0, \hat{x}<\infty$ and $a(1 /(1-q))=-\gamma\left(\hat{x}-F^{\leftarrow}(q)\right)$. From (4.1.4) and Lemma 4.1.2, we obtain as $t \rightarrow \infty$,

$$
\lim _{q \rightarrow 1} \frac{\hat{x}-\mathbb{E}\left[X \mid X>F^{\leftarrow}(q), Y>F^{\leftarrow}(q)\right]}{\hat{x}-F^{\leftarrow}(q)}=1+\gamma \int_{0}^{1} \frac{x^{-\gamma}-1}{\gamma} \mathrm{d} \tau(x, 1)=1-\int_{0}^{1} \tau\left(x^{-1 / \gamma}, 1\right) \mathrm{d} x .
$$

\subsection{Examples}

In this section, we first show that many commonly used copulas do satisfy Assumptions 3.1.1 and 4.1.1. Then with the MDA modelling for the marginal risks, we present the asymptotic expansions of the JES for these copulas.

\section{Independence survival copula}

The independence survival copula is $\widehat{C}(u, v)=u v$. Straightforwardly we have

$$
\frac{\widehat{C}(u t, v t)}{\widehat{C}(t, t)}=\frac{u t v t}{t^{2}}=u v:=\tau(u, v) .
$$

so that Assumption 3.1.1 is satisfied. Also by the definition (3.1.2), the tail order $\kappa$ is 2, and $\ell(t)=1$. Next we verify Assumption 4.1.1. Obviously, $\tau(x, 1)=x$ for all $0 \leq x \leq 1$, which implies $\beta=1$ and $h(x)=1$.

By Theorem 4.1.1, for $X$ with a distribution function $F$ and a tail quantile function $U \in \mathrm{ERV}_{\gamma}$ with $\gamma<1$, since the asymptotic expansions of the JES for the Gumbel case are not as informative as the other two cases, we only show the expansions for the Fréchet and Weibull cases: 
- Fréchet case $(\gamma>0)$ :

$$
\mathbb{E}\left[X \mid X>F^{\leftarrow}(q), Y>G^{\leftarrow}(q)\right] \sim \frac{1}{1-\gamma} F^{\leftarrow}(q) ;
$$

- Weibull case $(\gamma<0)$ :

$$
\hat{x}-\mathbb{E}\left[X \mid X>F^{\leftarrow}(q), Y>G^{\leftarrow}(q)\right] \sim \frac{1}{1-\gamma}\left(\hat{x}-F^{\leftarrow}(q)\right) .
$$

\section{Extreme value survival copula}

The extreme value survival copula is given by

$$
\widehat{C}(u, v)=\exp \{-A(-\log u,-\log v)\}
$$

where $A:[0, \infty)^{2} \rightarrow[0, \infty)$ is convex homogeneous of order 1 and satisfies $\max \{x, y\} \leq$ $A(x, y) \leq x+y$. Note that for $u, v \geq 0$, as $t \downarrow 0$, we have

$$
\begin{aligned}
\frac{\widehat{C}(u t, v t)}{\widehat{C}(t, t)} & =\frac{\exp (-A(-\log u t,-\log v t))}{\exp (A(1,1) \log t)} \\
& =\exp \left(\log t\left(A\left(\frac{\log u}{\log t}+1, \frac{\log v}{\log t}+1\right)-A(1,1)\right)\right) \\
& \rightarrow u^{A_{1}(1,1)} v^{A_{2}(1,1)}:=\tau(u, v) .
\end{aligned}
$$

Thus, Assumption 3.1.1 is satisfied. From (3.1.2), the tail order is $\kappa=A(1,1) \in[1,2]$ and $\ell(t)=1$. Now we check Assumption 4.1.1: for $0 \leq x \leq 1, \tau(x, 1)=x^{A_{1}(1,1)}$, which implies $\beta=A_{1}(1,1)$ and $h(x)=1$.

By Theorem 4.1.1, for $X$ with a distribution function $F$ and a tail quantile function $U \in \mathrm{ERV}_{\gamma}$ with $\gamma<A_{1}(1,1) \wedge 1$, we have

- Fréchet case $(\gamma>0)$ :

$$
\mathbb{E}\left[X \mid X>F^{\leftarrow}(q), Y>G^{\leftarrow}(q)\right] \sim \frac{A_{1}(1,1)}{A_{1}(1,1)-\gamma} F^{\leftarrow}(q) ;
$$


- Weibull case $(\gamma<0)$ :

$$
\hat{x}-\mathbb{E}\left[X \mid X>F^{\leftarrow}(q), Y>G^{\leftarrow}(q)\right] \sim \frac{A_{1}(1,1)}{A_{1}(1,1)-\gamma}\left(\hat{x}-F^{\leftarrow}(q)\right) .
$$

If $A$ is symmetric, then by Euler's theorem, for homogenous function $A(1,1)=A_{1}(1,1)+$ $A_{2}(1,1)$. It implies that $A_{1}(1,1)=A(1,1) / 2$. Thus, $\beta=\kappa / 2$. In this case, the stronger dependence $($ smaller $\kappa$ ) will result in increase of JES.

\section{Archimedean survival copula}

Archimedean survival copula is given by

$$
\widehat{C}(u, v)=\psi\left(\psi^{-1}(u)+\psi^{-1}(v)\right),
$$

where $\psi(\infty)=0, \psi(0)=1$, and $\psi$ is 2-times monotone (non-increasing and convex). Assume that $\psi \in \mathrm{RV}_{-\alpha}$, with $\alpha>0$. By Proposition B.1.9 of Bingham et al. (1989), $\psi^{-1} \in \mathrm{RV}_{-1 / \alpha}$, we have

$$
\frac{\widehat{C}(u t, v t)}{\widehat{C}(t, t)}=\frac{\psi\left(\psi^{-1}(u t)+\psi^{-1}(v t)\right)}{\psi\left(2 \psi^{-1}(t)\right)} \rightarrow 2^{\alpha}\left(u^{-\frac{1}{\alpha}}+v^{-\frac{1}{\alpha}}\right)^{-\alpha}:=\tau(u, v) .
$$

Hence, Assumption 3.1.1 is satisfied. From (3.1.2), note that $t=\psi\left(\psi^{-1}(t)\right)$, we have the tail order is $\kappa=1$ and $\ell(t)=1$. Now we check Assumption 4.1.1. First note that as $x \rightarrow 0$,

$$
\frac{\tau(x y, 1)}{\tau(x, 1)}=\frac{2^{\alpha}\left((x y)^{-\frac{1}{\alpha}}+1\right)^{-\alpha}}{2^{\alpha}\left(x^{-\frac{1}{\alpha}}+1\right)^{-\alpha}}=\left(\frac{(x y)^{-\frac{1}{\alpha}}+1}{x^{-\frac{1}{\alpha}}+1}\right)^{-\alpha} \rightarrow y .
$$

Thus, $\tau(\cdot, 1) \in \operatorname{RV}_{1}(0+)$, which means $\beta=1$. The slowly varying function for $\tau(x, 1)$ is

$$
h(x)=\frac{\tau(x, 1)}{x}=2^{\alpha}\left(1+x^{\frac{1}{\alpha}}\right)^{-\alpha} \leq 2^{\alpha}
$$

for $0 \leq x \leq 1$. Thus, $\tau(x, 1) \leq 2^{\alpha} x$ for $0 \leq x \leq 1$, and Assumption 4.1.1 is satisfied. 
By Theorem 4.1.1, for $X$ with a distribution function $F$ and a tail quantile function $U \in \mathrm{ERV}_{\gamma}$ with $\gamma<1$, we have

- Fréchet case $(\gamma>0)$ :

$$
\mathbb{E}\left[X \mid X>F^{\leftarrow}(q), Y>G^{\leftarrow}(q)\right] \sim F^{\leftarrow}(q)\left(1+2^{\alpha} \int_{1}^{\infty}\left(x^{\frac{1}{\alpha \gamma}}+1\right)^{-\alpha} \mathrm{d} x\right) ;
$$

- Weibull case $(\gamma<0)$ :

$$
\hat{x}-\mathbb{E}\left[X \mid X>F^{\leftarrow}(q), Y>G^{\leftarrow}(q)\right] \sim\left(\hat{x}-F^{\leftarrow}(q)\right)\left(1-2^{\alpha} \int_{0}^{1}\left(x^{\frac{1}{\alpha \gamma}}+1\right)^{-\alpha} \mathrm{d} x\right) .
$$

\subsection{A Generalization}

We conclude this chapter by considering a further generalization of JES, i.e.

$$
\mathbb{E}\left[X \mid X>\zeta F^{\leftarrow}(q), Y>G^{\leftarrow}(q)\right]
$$

where $\zeta$ is a constant between 0 and 1 . This measure provides an additional flexibility that the given information of $X$ and $Y$ are large but not at the same scale. When $\zeta$ approaches 1 , then (4.3.1) recovers the original definition of JES. When $\zeta$ is close to 0 , then it behaves more like MES. For this reason, we refer (4.3.1) as the general JES.

For simplicity and illustration purpose, we only show the asymptotic expansion of the general JES for the Fréchet case $(\gamma>0)$ in the next theorem.

Theorem 4.3.1 Suppose that $X$ follows a distribution function $F$ with a tail quantile function $U \in \operatorname{ERV}_{\gamma}$ with $0<\gamma<1 \wedge \beta$ and auxiliary function $a(\cdot)$, and $Y$ follows a distribution function $G$. Let $\widehat{C}$ be the survival copula of $(X, Y)$ satisfying Assumptions 3.1.1 and 4.1.1. Then we have for $0<\zeta \leq 1$, as $q \uparrow 1$

$$
\mathbb{E}\left[X \mid X>\zeta F^{\leftarrow}(q), Y>G^{\leftarrow}(q)\right] \sim F^{\leftarrow}(q)\left(\zeta+\frac{1}{\tau\left(\zeta^{-1 / \gamma}, 1\right)} \int_{\zeta}^{\infty} \tau\left(x^{-1 / \gamma}, 1\right) \mathrm{d} x\right) .
$$


Proof. Follow the same setup as in the proof of Theorem 4.1.1. That is, let $V$ and $W$ be two uniformly distributed random variables on $(0,1)$. Then, $X \stackrel{d}{=} F^{\leftarrow}(1-V)=U\left(\frac{1}{V}\right)$, and $Y \stackrel{d}{=} G^{\leftarrow}(1-W)$. Letting $t=\frac{1}{1-q}$ results in

$$
\begin{aligned}
& \mathbb{E}\left[X \mid X>\zeta F^{\leftarrow}(q), Y>G^{\leftarrow}(q)\right]=\mathbb{E}\left[U\left(\frac{1}{V}\right) \mid V<\bar{F}\left(\zeta F^{\leftarrow}(1-1 / t)\right), W<\frac{1}{t}\right] \\
& =\frac{1}{\widehat{C}\left(\bar{F}\left(\zeta F^{\leftarrow}(1-1 / t)\right), 1 / t\right)} \int_{0}^{\bar{F}\left(\zeta F^{\leftarrow}(1-1 / t)\right)} U\left(\frac{1}{v}\right) \mathrm{d} \widehat{C}(v, 1 / t) \\
& =\frac{\widehat{C}(1 / t, 1 / t)}{\widehat{C}\left(\bar{F}\left(\zeta F^{\leftarrow}(1-1 / t)\right), 1 / t\right)} \frac{U(t)}{\widehat{C}(1 / t, 1 / t)} \int_{0}^{M} \frac{U\left(\frac{t}{x}\right)}{U(t)} 1_{\left\{x \leq \bar{F}\left(\zeta F^{\leftarrow}(1-1 / t)\right) t\right\}} \mathrm{d} \widehat{C}\left(\frac{x}{t}, \frac{1}{t}\right) \\
& \leq \frac{\widehat{C}(1 / t, 1 / t)}{\widehat{C}\left(\bar{F}\left(\zeta F^{\leftarrow}(1-1 / t)\right), 1 / t\right)} \frac{U(t)}{\widehat{C}(1 / t, 1 / t)} \int_{0}^{M} \frac{U\left(\frac{t}{x}\right)}{U(t)} \mathrm{d} \widehat{C}\left(\frac{x}{t}, \frac{1}{t}\right),
\end{aligned}
$$

where the third step is due to that given $\zeta$, there exists $M>0$ such that when $t$ is large enough, $\bar{F}\left(\zeta F^{\leftarrow}(1-1 / t)\right) t<M$. In fact, due to that $\bar{F} \in \mathrm{RV}_{-1 / \gamma}$ and $F^{\leftarrow} \in \mathrm{RV}_{-\gamma}(1-)$, we have for any $\varepsilon>0$,

$$
\bar{F}\left(F^{\leftarrow}(1-1 / t)(1+\varepsilon)\right) \leq \frac{1}{t} \leq \bar{F}\left(F^{\leftarrow}(1-1 / t)(1-\varepsilon)\right) .
$$

Thus,

$$
\lim _{t \rightarrow \infty} \bar{F}\left(F^{\leftarrow}(1-1 / t)\right) t=1
$$

and

$$
\lim _{t \rightarrow \infty} \bar{F}\left(\zeta F^{\leftarrow}(1-1 / t)\right) t=\lim _{t \rightarrow \infty} \frac{\bar{F}\left(\zeta F^{\leftarrow}(1-1 / t)\right)}{\bar{F}\left(F^{\leftarrow}(1-1 / t)\right)}=\zeta^{-1 / \gamma} .
$$

Further, by Potter's bound, for $0<\zeta<1$, there exists $M>0$ such that

$$
\bar{F}\left(\zeta F^{\leftarrow}(1-1 / t)\right) t \leq M
$$

Also note that

$$
\lim _{t \rightarrow \infty} \frac{\widehat{C}(1 / t, 1 / t)}{\widehat{C}\left(\bar{F}\left(\zeta F^{\leftarrow}(1-1 / t)\right), 1 / t\right)}=\frac{1}{\tau\left(\zeta^{-1 / \gamma}, 1\right)} .
$$


Using similar arguments as in the proof of Lemma 4.1.2, we know that $\frac{1}{\widehat{C}(1 / t, 1 / t)} \int_{0}^{M} \frac{U\left(\frac{t}{x}\right)}{U(t)} \mathrm{d} \widehat{C}\left(\frac{x}{t}, \frac{1}{t}\right)$ in (4.3.3) is integrable. Then by the dominated convergence theorem the desired result follows. 


\section{Chapter 5}

\section{Interplay of Dependence and Heavy Tailedness}

It is known that the crude Monte Carlo simulation is very inefficient for the study of rare events, especially when it comes to the multidimensional case. The asymptotic analysis in Chapter 4 provides a way to investigate the extreme risks in the 2-dimensional case so that the effects of dependence and heavy tailedness can be easily explored. In this chapter, through concrete examples, we discuss how the dependence and heavy tailedness affect the measure of extreme risks. All the calculations of ES, MES and JES are based on their asymptotic expansions.

\subsection{A Numerical Example of Gumbel Survival Copula}

First, we focus on the asymptotic independence structure, that is when $1<\kappa \leq 2$. Assume that $X$ and $Y$ follow a Gumbel survival copula; i.e.

$$
\widehat{C}(u, v)=\exp \left\{-\left((-\log u)^{\theta}+(-\log v)^{\theta}\right)^{1 / \theta}\right\}
$$


where $\theta \geq 1$. Gumbel copula belongs to the extreme value copula class with a homogeneous function $A(u, v)=\left(u^{\theta}+v^{\theta}\right)^{\frac{1}{\theta}}$. One can easily check that the Gumbel survival copula satisfies Assumption 3.1.1 with $\tau(u, v)=(u v)^{2^{1 / \theta-1}}$ and the tail order $\kappa=2^{\frac{1}{\theta}}$. Moreover, from Assumption 4.1.1, $\beta=2^{\frac{1}{\theta}-1}$. Suppose $X$ follows a Pareto distribution for some $\gamma>0$

$$
F(x)=1-\left(\frac{1}{x+1}\right)^{1 / \gamma}, \quad x>0
$$

It can be showed that the tail quantile function of $X$ is ERV with index of $\gamma>0$.

In Figure 5.1 we plot the JES of $X$ and $Y$ using the result from Theorem 4.1 .1 (i). When $\kappa=2, \gamma$ is taken to be $0.1,0.5$ and 0.9 . When $\kappa=1.5$, due to the constraint that $\gamma<1 \wedge \beta$, we choose $\gamma=0.1$ and 0.5. Because the range of values for JES is too large, we plot the log of JES in Figure 5.1. As expected, under the same dependence or heavy tailedness, the heavier tailed marginal (bigger $\gamma$ ) or stronger dependence (lower $\kappa)$ yields a higher risk. An interesting observation is that the heavy tailedness of $X$ is a more significant driver of the risk than the dependence. This shows that when an extreme event is observed in $Y$, the dependence between $X$ and $Y$ indeed increases the risk of $X$ to have potentially bigger loss though the level of riskiness is still determined by the heavy tailedness of $X$.

\subsection{A Numerical Example of Clayton Survival Copula}

We next consider the effect of the asymptotic dependence structure on quantifying extreme risks. The Clayton survival copula is a special case of Archimedean copula with the following generator function

$$
\psi(t)=(1+t)^{-\alpha}
$$

In our example we take $\alpha=1$. We similarly assume that both $X$ and $Y$ follow a Pareto distribution with $\gamma=0.6$. In this case, we calculate JES with $\zeta=0.3,0.7$ and 1 using (4.3.2) in Theorem 4.3.1. The results are plotted in Figure 5.2 against the values of $q$ over 


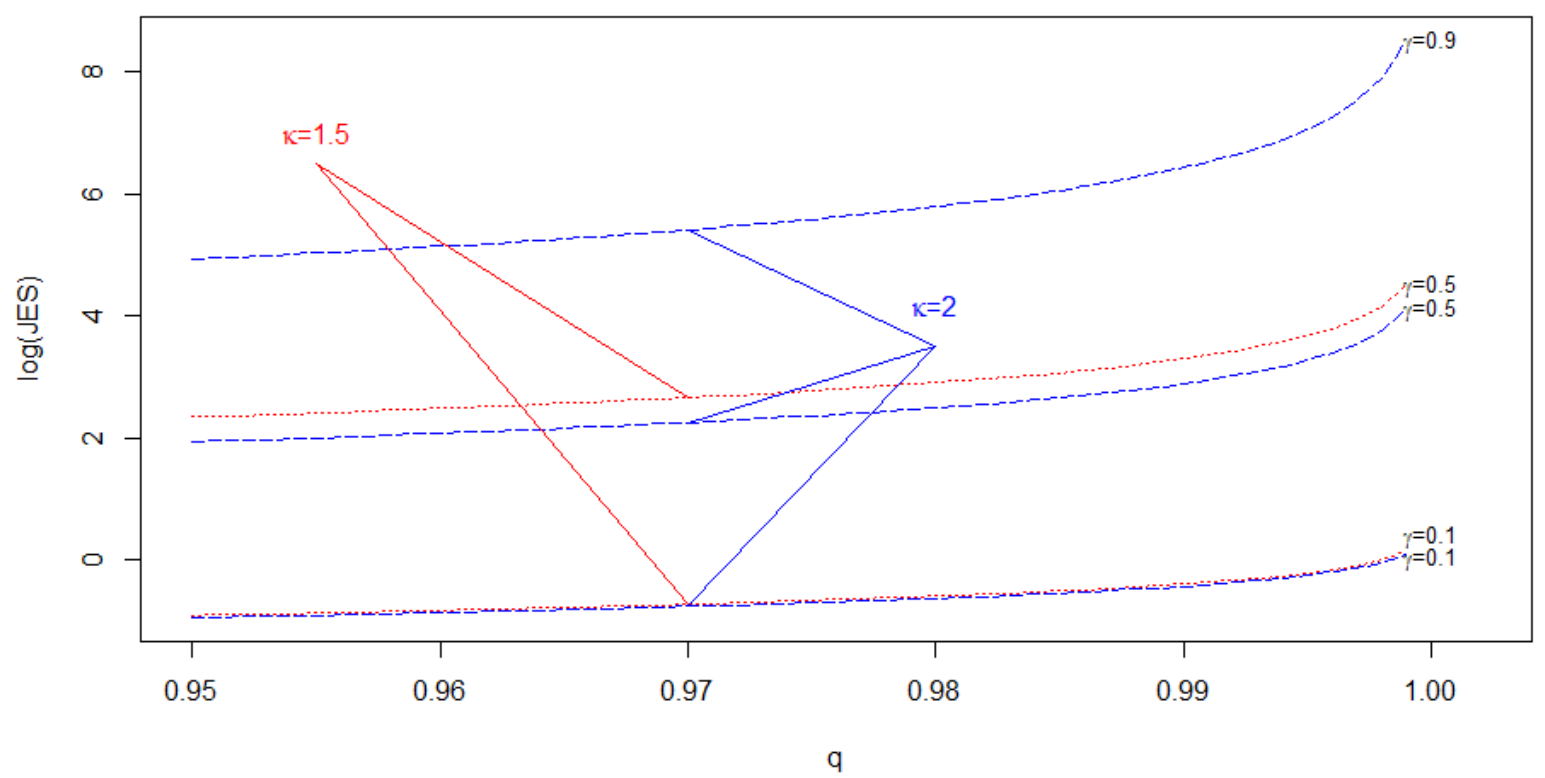

Figure 5.1: From $q=0.95$ to $q=0.999$, the $\log$ of JES is plotted. $X$ and $Y$ are coupled by a Gumbel survival copula with $\kappa=1.5$ and 2. $X$ is Pareto distributed with $\gamma=0.1,0.5$ and 0.9 .

the range $(0.985,0.999)$. Recall that for the special case $\zeta=0$, the general JES reduces to MES and we similarly plot its values based on the asymptotic expansion obtained from Proposition 10 of Hua and Joe (2014). To provide more insights for the comparisons, we also plot ES using the asymptotic expansions derived in Appendix A. Note that for a given value of $q$, the general JES is bounded from above when $\zeta=1$ (i.e. JES) and from below when $\zeta=0$ (i.e. MES). Hence as $\zeta$ decreases, so is the general JES. This should not be surprising as one observes that $X$ is not in its far tail as $Y$, the risk of $X$ should be smaller. The value of ES lies between MES and JES. MES only uses the information of $Y$, which means it exploits the dependence between $X$ and $Y$. This further implies that $Y$ is not able to fully capture the information whether $X$ is in its extreme, unless $X$ and $Y$ are comonotonic. ES uses the information of $X$ solely; i.e. without considering the dependence. The fact that ES is larger than MES provides an indication that heavy tailedness plays a 
more dominant role than dependence. Finally, the measure of JES takes a step further by exploiting the extremes of both $X$ and $Y$. By doing so JES is larger than ES, highlighting dependence also contributes to risk. Hence it is of paramount importance to take into consideration both heavy tailedness and dependence jointly. Overlooking either of these attributes is undesirable and understates the underlying risk, as in both ES and MES.

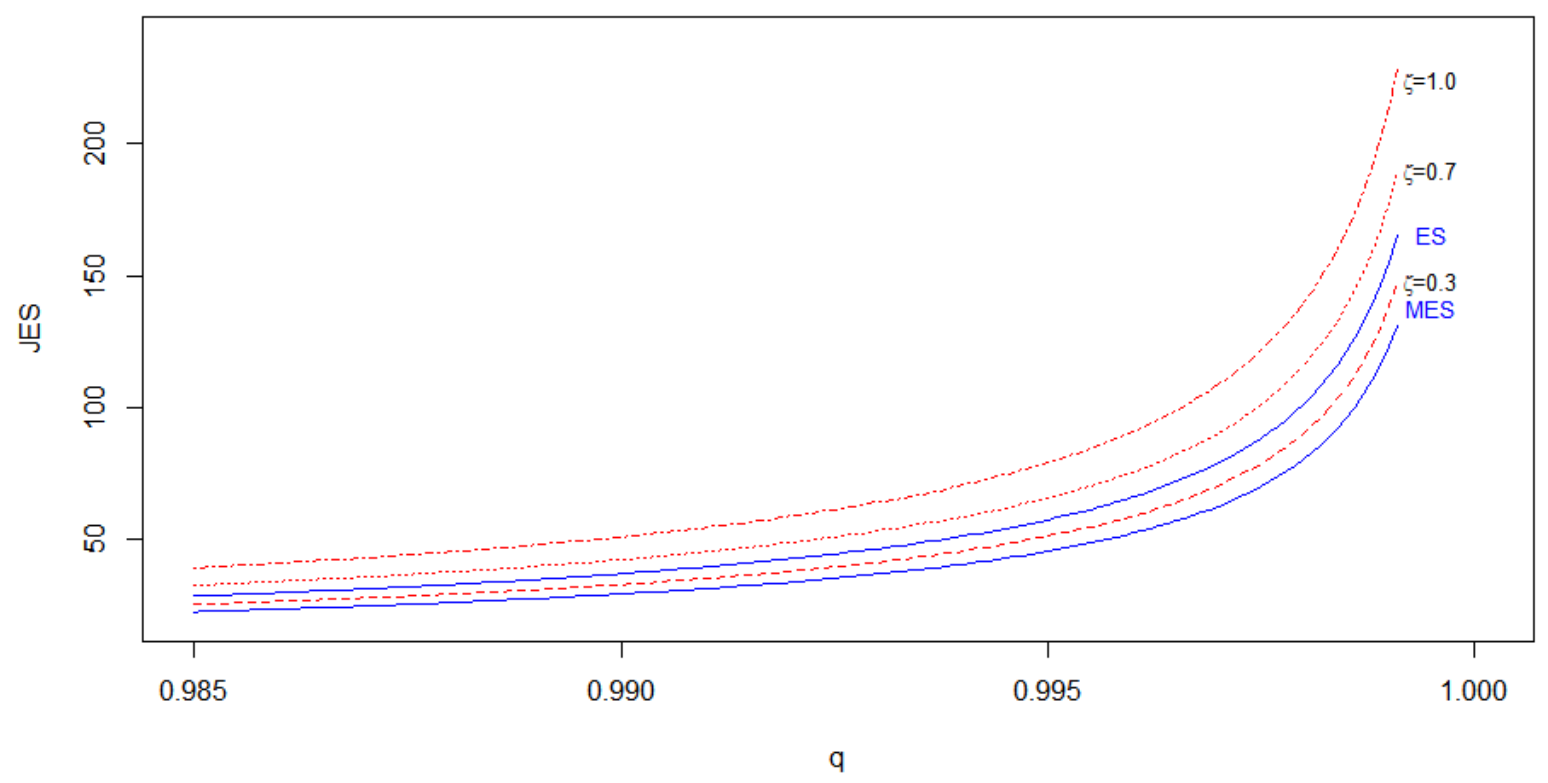

Figure 5.2: From $q=0.985$ to $q=0.999, \mathbb{E}\left[X \mid X>\zeta F^{\leftarrow}(q), Y>G^{\leftarrow}(q)\right]$ is plotted with $\zeta=0.3$, 0.7 , and 1. Both MES and ES are plotted for comparison. $X$ and $Y$ follow a Pareto distribution with $\gamma=0.6$ and are coupled by the Clayton survival copula with $\alpha=1$.

As noted above, measures such as ES and MES that ignore the joint effect of dependence and heavy tailedness may underestimate the underlying risk. We explore this issue further. First we consider the case with asymptotic independence structure. Again we assume $X$ and $Y$ follow a Gumbel survival copula and $X$ is Pareto distributed. Table 5.1 displays the ratio of JES and ES for different level of dependence and heavy tailedness. Due to the restriction of $\gamma<1 \wedge \beta$, the lower triangle of the table is blank. From the reported ratios, 
we draw the following key observations. First of all, when $\kappa=2$, which is the independence structure, JES and ES are the same as expected. As long as $X$ is less heavy tail (such as $\gamma=0.2$ ), both JES and ES are close to each other, irrespective of the dependence. However, as the dependence becomes stronger and the tail is heavier, the combined effect has a greater effect on JES than on ES, as exemplified by the much larger value of JES relative to ES. More specifically, when there is a much stronger dependence, JES can be more than 5 times larger than the corresponding ES. These observations signify that the joint effect of dependence and heavy tailedness has a more profound effect on risk. This, in turn, also unequivocally points out the limitation of ES. By overlooking dependence, especially when there is systemic risk in the market, the use of ES can dramatically underestimate the underlying risk.

\begin{tabular}{cccccccc}
\hline \hline \multirow{2}{*}{ Tail order $\kappa$} & \multicolumn{7}{c}{ Tail index $\gamma$} \\
\cline { 2 - 7 } & 0.9 & 0.8 & 0.7 & 0.6 & 0.5 & 0.4 & 0.2 \\
\hline 2 & 1.000 & 1.000 & 1.000 & 1.000 & 1.000 & 1.000 & 1.000 \\
1.9 & 1.900 & 1.267 & 1.140 & 1.086 & 1.056 & 1.036 & 1.013 \\
1.8 & & 1.800 & 1.350 & 1.200 & 1.125 & 1.080 & 1.029 \\
1.7 & & 3.400 & 1.700 & 1.360 & 1.214 & 1.133 & 1.046 \\
1.6 & & & 2.400 & 1.600 & 1.333 & 1.200 & 1.067 \\
1.5 & & 4.500 & 2.000 & 1.500 & 1.286 & 1.091 \\
1.4 & & & 2.800 & 1.750 & 1.400 & 1.120 \\
1.3 & & & 5.200 & 2.167 & 1.560 & 1.156 \\
1.2 & & & & 3.000 & 1.800 & 1.200 \\
1.1 & & & & 5.500 & 2.200 & 1.257 \\
\hline \hline
\end{tabular}

Table 5.1: The ratio of JES and ES. $X$ and $Y$ follow a Gumbel survival copula. $X$ is Pareto distributed.

Next we consider the asymptotic dependence structure by letting $X$ and $Y$ follow a Clayton survival copula, while still letting $X$ be Pareto distributed. Because the Clayton survival copula has $\kappa=1$, we apply the tail dependence coefficient $\lambda$ to further classify the asymptotic dependence case. That is,

$$
\lambda=\lim _{q \rightarrow 1} \operatorname{Pr}\left(X>F^{\rightarrow}(q) \mid Y>G^{\leftarrow}(q)\right)
$$


see for example McNeil et al. (2015). It is easy to verify that $\lambda=2^{-\alpha}$ for the Clayton survival copula. Table 5.2 provides similar comparison as in Table 5.1 except the dependence of $X$ and $Y$ is measured by the tail dependence coefficient. First note that in one extreme, when $X$ and $Y$ are highly dependent (such as $\lambda=0.9$ ), both JES and ES are found to be close to each other, regardless of the tail heaviness. This is to be expected since the information provided by the extremes of $X$ and $Y$ overlaps significantly. Second, in another extreme when $X$ is lighter tailed (such as $\gamma=0.1$ ), both JES and ES are close to each other, irrespective of dependence. Third and most importantly, as dependence gets weaker (but still asymptotically dependent), the heavy tailedness becomes more prominent in the sense that JES is progressively (much) larger than the corresponding ES with increasing heavy tailedness of $X$. This phenomenon again signifies the importance of taking into consideration both heavy tailedness and dependence. ES that only reflects the tail heaviness of $X$ can severely understate the underlying risk. In our example, the severity of the understatement relative to JES can be as high as almost 6 times. Finally, recall that the ratios in Table 5.1 refer to cases with asymptotic independence structure while the ratios in Table 5.2 are for cases with asymptotic dependence structure. For a given level of dependence, the ratio of JES to ES in both cases increases with heavy tailedness. If the heavy tailedness of $X$ is held constant, the dependence has an opposite effect on the underlying risk. In particular, as dependence gets stronger, the ratio in the former case (with asymptotic independence structure) increases while the ratio in the latter case (with asymptotic dependence structure) decreases.

Continue with the same example as Table 5.2, Table 5.3 provides a similar comparison except it tabulates the ratio of JES to MES. The observations we made in Table 5.2 similarly apply to Table 5.3 but for MES. For example, MES severely understates the underlying risk under the joint conditions that $X$ is heavy tailedness and weaker dependence (but still asymptotically dependent) between $X$ and $Y$. In some cases, the degree of the understatement is almost 10 times as large. Note also that the ratios in Table 5.3 are larger than the corresponding ratios in Table 5.2. This is consistent with our observation in Figure 5.2 that ES lies between MES and JES. By comparing Table 5.2 to Table 5.3, it leads to the conclusion that between dependence and heavy tailedness, it is relatively 


\begin{tabular}{ccccccc}
\hline \hline \multirow{2}{*}{ Tail dependence coefficient $\lambda$} & \multicolumn{7}{c}{ Tail index $\gamma$} \\
\cline { 2 - 7 } & 0.9 & 0.8 & 0.6 & 0.4 & 0.2 & 0.1 \\
\hline 0.1 & 5.726 & 3.758 & 2.111 & 1.477 & 1.171 & 1.074 \\
0.2 & 3.543 & 2.695 & 1.808 & 1.381 & 1.145 & 1.064 \\
0.3 & 2.641 & 2.179 & 1.623 & 1.314 & 1.125 & 1.056 \\
0.4 & 2.127 & 1.853 & 1.486 & 1.258 & 1.106 & 1.049 \\
0.5 & 1.789 & 1.621 & 1.377 & 1.209 & 1.089 & 1.041 \\
0.6 & 1.546 & 1.444 & 1.284 & 1.164 & 1.072 & 1.034 \\
0.7 & 1.362 & 1.302 & 1.202 & 1.121 & 1.055 & 1.026 \\
0.8 & 1.216 & 1.185 & 1.129 & 1.080 & 1.038 & 1.018 \\
0.9 & 1.098 & 1.086 & 1.062 & 1.040 & 1.019 & 1.010 \\
\hline \hline
\end{tabular}

Table 5.2: The ratio of JES and ES. $\mathrm{X}$ and $\mathrm{Y}$ follow a Clayton survival copula. $\mathrm{X}$ is Pareto distributed.

more important to capture the heavy tailedness. Ideally, one should adopt a risk measure that takes into consideration both of these effects jointly, as in JES.

\begin{tabular}{ccccccc}
\hline \hline \multirow{2}{*}{ Tail dependence coefficient $\lambda$} & \multicolumn{7}{c}{ Tail index $\gamma$} \\
\cline { 2 - 7 } & 0.9 & 0.8 & 0.6 & 0.4 & 0.2 & 0.1 \\
\hline 0.1 & 9.872 & 9.565 & 8.158 & 5.707 & 2.981 & 1.852 \\
0.2 & 4.911 & 4.745 & 4.129 & 3.155 & 2.016 & 1.475 \\
0.3 & 3.264 & 3.153 & 2.793 & 2.261 & 1.627 & 1.305 \\
0.4 & 2.444 & 2.364 & 2.130 & 1.803 & 1.411 & 1.205 \\
0.5 & 1.955 & 1.896 & 1.736 & 1.525 & 1.273 & 1.138 \\
0.6 & 1.631 & 1.587 & 1.478 & 1.340 & 1.179 & 1.091 \\
0.7 & 1.402 & 1.371 & 1.298 & 1.210 & 1.110 & 1.056 \\
0.8 & 1.232 & 1.212 & 1.167 & 1.117 & 1.061 & 1.031 \\
0.9 & 1.102 & 1.092 & 1.071 & 1.049 & 1.025 & 1.013 \\
\hline \hline
\end{tabular}

Table 5.3: The ratio of JES and MES. X and Y follow a Clayton survival copula. X and Y are Pareto distributed. 


\section{Chapter 6}

\section{Conclusion}

We study the effects of tail dependence and heavy tailedness through quantifying extreme risks. The tail dependence is investigated through regularly varying copulas, which is known as the copulas having tail order property in the literature. Tail orders can better capture tail independence structures, compared to the tail dependence coefficient. Copulas with tail order property are characterized through multivariate regular variation. A uniform convergence property of these copulas are constructed in parallel to that of the univariate regularly varying function. The JES is proposed to measure the multivariate extreme risks. Asymptotic analysis of the JES is carried out to help the study of interplay between tail dependence and heavy tailedness. We find that overall dependence plays an important role in the measure of extreme risks. In the tail dependent case, the heavy tailedness is a much stronger driving factor than the dependence. Comparing to ES, the JES takes dependence into accounts to avoid the underestimation of risks. Comparing to MES, the JES takes more of the heavy tailedness into consideration to avoid the underestimation of risk, and it can easily evaluate risks under all dependence structures. 


\section{References}

Acharya, V. V., Pedersen, L. H., Philippon, T., and Richardson, M. (2017). Measuring systemic risk. The Review of Financial Studies, 30(1):2-47.

Adrian, T. and Brunnermeier, M. K. (2011). Covar. Technical report, National Bureau of Economic Research.

Artzner, P., Delbaen, F., Eber, J.-M., and Heath, D. (1999). Coherent measures of risk. Mathematical finance, 9(3):203-228.

Asimit, A. V., Furman, E., Tang, Q., and Vernic, R. (2011). Asymptotics for risk capital allocations based on conditional tail expectation. Insurance: Mathematics \& Economics, $49(3): 310-324$.

Asimit, A. V. and Jones, B. L. (2008). Asymptotic tail probabilities for large claims reinsurance of a portfolio of dependent risks. Astin Bulletin. The Journal of the International Actuarial Association, 38(1):147-159.

Bargès, M., Cossette, H., and Marceau, E. (2009). Tvar-based capital allocation with copulas. Insurance: Mathematics and Economics, 45(3):348-361.

Bernard, C. and Czado, C. (2015). Conditional quantiles and tail dependence. Journal of Multivariate Analysis, 138:104-126.

Bingham, N. H., Goldie, C. M., and Teugels, J. L. (1989). Regular variation, volume 27 of Encyclopedia of Mathematics and its Applications. Cambridge University Press, Cambridge. 
Cai, J. and Li, H. (2005). Conditional tail expectations for multivariate phase-type distributions. Journal of Applied Probability, 42(3):810-825.

Cai, J.-J., Einmahl, J. H. J., de Haan, L., and Zhou, C. (2015). Estimation of the marginal expected shortfall: the mean when a related variable is extreme. Journal of the Royal Statistical Society. Series B. Statistical Methodology, 77(2):417-442.

de Haan, L. and Ferreira, A. (2006). Extreme value theory. Springer Series in Operations Research and Financial Engineering. Springer, New York. An introduction.

Drees, H. (1998). On smooth statistical tail functionals. Scandinavian Journal of Statistics. Theory and Applications, 25(1):187-210.

Embrechts, P., Klüppelberg, C., and Mikosch, T. (1997). Modelling extremal events, volume 33 of Applications of Mathematics (New York). Springer-Verlag, Berlin. For insurance and finance.

Embrechts, P., McNeil, A., and Straumann, D. (2002). Correlation and dependence in risk management: properties and pitfalls. Risk management: value at risk and beyond, $1: 176-223$.

Fisher, R. A. and Tippett, L. H. C. (1928). Limiting forms of the frequency distribution of the largest or smallest member of a sample. In Mathematical Proceedings of the Cambridge Philosophical Society, volume 24, pages 180-190. Cambridge University Press.

Gabaix, X. (2009). Power laws in economics and finance. Annual Review of Economics, $1(1): 255-294$.

Gabaix, X., Gopikrishnan, P., Plerou, V., and Stanley, H. E. (2006). Institutional investors and stock market volatility. The Quarterly Journal of Economics, 121(2):461-504.

Glasserman, P. (2004). Monte Carlo methods in financial engineering, volume 53 of Applications of Mathematics (New York). Springer-Verlag, New York. Stochastic Modelling and Applied Probability. 
Gnedenko, B. (1943). Sur la distribution limite du terme maximum d'une serie aleatoire. Annals of mathematics, pages 423-453.

Gudendorf, G. and Segers, J. (2010). Extreme-value copulas. In Copula theory and its applications, volume 198 of Lect. Notes Stat. Proc., pages 127-145. Springer, Heidelberg.

Guillaume, D. M., Dacorogna, M. M., Davé, R. R., Müller, U. A., Olsen, R. B., and Pictet, O. V. (1997). From the bird's eye to the microscope: A survey of new stylized facts of the intra-daily foreign exchange markets. Finance and stochastics, 1(2):95-129.

Hua, L. and Joe, H. (2011). Tail order and intermediate tail dependence of multivariate copulas. Journal of Multivariate Analysis, 102(10):1454-1471.

Hua, L. and Joe, H. (2014). Strength of tail dependence based on conditional tail expectation. Journal of Multivariate Analysis, 123:143-159.

Jiang, C. and Long, W. (2018). Does tail dependence make a difference in the estimation of systematic risk?: $\Delta$ covar and mes. Available at SSRN 3296750.

Joe, H. and Li, H. (2011). Tail risk of multivariate regular variation. Methodology and Computing in Applied Probability, 13(4):671-693.

Landsman, Z. M. and Valdez, E. A. (2003). Tail conditional expectations for elliptical distributions. North American Actuarial Journal, 7(4):55-71.

McNeil, A. J., Frey, R., and Embrechts, P. (2015). Quantitative Risk Management: Concepts, Techniques and Tools-revised edition. Princeton university press.

Nelsen, R. B. (2006). An introduction to copulas. Springer Series in Statistics. Springer, New York, second edition.

Nešlehová, J., Embrechts, P., and Chavez-Demoulin, V. (2006). Infinite mean models and the lda for operational risk. Journal of Operational Risk, 1(1):3-25.

Resnick, S. I. (2007). Heavy-tail phenomena: probabilistic and statistical modeling. Springer Science \& Business Media. 
Schmidt, R. and Stadtmüller, U. (2006). Non-parametric estimation of tail dependence. Scandinavian Journal of Statistics, 33(2):307-335.

Vernic, R. (2006). Multivariate skew-normal distributions with applications in insurance. Insurance: Mathematics and economics, 38(2):413-426.

Zhu, L. and Li, H. (2012). Asymptotic analysis of multivariate tail conditional expectations. North American Actuarial Journal, 16(3):350-363. 
APPENDICES 


\section{Appendix A}

\section{Asymptotic Expansions of ES}

Although the asymptotic expansion of ES when $X$ is regularly varying is well known in the literature, it is usually presented as a special case of some general risk measures. For completeness, we drive the asymptotic expansions of ES when $X$ belongs to a MDA.

Proposition A.0.1 Suppose that $X$ follows a distribution function $F$ with a tail quantile function $U \in \mathrm{ERV}_{\gamma}$ with $\gamma<1$ and auxiliary function $a(\cdot)$. Then we have as $q \uparrow 1$

(i) Fréchet case $(\gamma>0)$ :

$$
\mathbb{E}\left[X \mid X>F^{\leftarrow}(q)\right] \sim \frac{1}{1-\gamma} F^{\leftarrow}(q)
$$

(ii) Gumbel case $(\gamma=0)$ : if $\hat{x}=\infty$, then

$$
\mathbb{E}\left[X \mid X>F^{\leftarrow}(q)\right] \sim F^{\leftarrow}(q),
$$

if $\hat{x}<\infty$, then

$$
\hat{x}-\mathbb{E}\left[X \mid X>F^{\leftarrow}(q)\right] \sim \hat{x}-F^{\leftarrow}(q) ;
$$


(iii) Weibull case $(\gamma<0)$ :

$$
\hat{x}-\mathbb{E}\left[X \mid X>F^{\leftarrow}(q)\right] \sim \frac{1}{1-\gamma}\left(\hat{x}-F^{\leftarrow}(q)\right)
$$

Proof. Note the rewriting

$$
\mathbb{E}\left[X \mid X>F^{\leftarrow}(q)\right]=a(t) \int_{0}^{1} \frac{U(t / x)-U(t)}{a(t)} \mathrm{d} x+U(t) .
$$

In view of (4.1.2), since $\gamma<1$, by the dominated convergence theorem, we have

$$
\lim _{t \rightarrow \infty} \int_{0}^{1} \frac{U(t / x)-U(t)}{a(t)} \mathrm{d} x=\int_{0}^{1} \frac{x^{-\gamma}-1}{\gamma} \mathrm{d} x .
$$

The rest of the proof is similar to that of Theorem 4.1.1. 


\section{Appendix B}

\section{Numerical Example of Clayton Copula}

For Clayton survival copula, the generator function is:

$$
\psi(t)=(1+t)^{-\alpha} \in R V_{-\alpha}, \alpha>0,
$$

and its corresponding copula function is

$$
\widehat{C}(u, v)=\psi\left(\psi^{-1}(u)+\psi^{-1}(v)\right)=\left(u^{-\frac{1}{\alpha}}+v^{-\frac{1}{\alpha}}-1\right)^{-\alpha} .
$$


The tail function defined on 3.1.2 can be calculated as:

$$
\begin{aligned}
b(u, v) & =\lim _{t \downarrow 0} \frac{\widehat{C}(u t, v t)}{t l(t)}=\lim _{t \downarrow 0} \frac{\left((u t)^{-\frac{1}{\alpha}}+(v t)^{-\frac{1}{\alpha}}-1\right)^{-\alpha}}{t l(t)} \\
& =\lim _{t \downarrow 0} \frac{\left((u t)^{-\frac{1}{\alpha}}+(v t)^{-\frac{1}{\alpha}}-1\right)^{-\alpha}}{\left(t^{-\frac{1}{\alpha}}\right)^{-\alpha}} \\
& =\lim _{t \downarrow 0}\left(\frac{(u t)^{-\frac{1}{\alpha}}+(v t)^{-\frac{1}{\alpha}}-1}{t^{-\frac{1}{\alpha}}}\right)^{-\alpha} \\
& =\lim _{t \downarrow 0}\left((u)^{-\frac{1}{\alpha}}+(v)^{-\frac{1}{\alpha}}-t^{\frac{1}{\alpha}}\right)^{-\alpha} \\
& =\left((u)^{-\frac{1}{\alpha}}+(v)^{-\frac{1}{\alpha}}\right)^{-\alpha} .
\end{aligned}
$$

The equivalent multivariate regular varying function stated on Assumption 3.1.1 is derived as:

$$
\begin{aligned}
\tau(u, v) & =\lim _{t \downarrow 0} \frac{\widehat{C}(u t, v t)}{\widehat{C}(t, t)}=\lim _{t \downarrow 0} \frac{\left((u t)^{-\frac{1}{\alpha}}+(v t)^{-\frac{1}{\alpha}}-1\right)^{-\alpha}}{\left((t)^{-\frac{1}{\alpha}}+(t)^{-\frac{1}{\alpha}}-1\right)^{-\alpha}} \\
& =\lim _{t \downarrow 0}\left(\frac{(u t)^{-\frac{1}{\alpha}}+(v t)^{-\frac{1}{\alpha}}-1}{2(t)^{-\frac{1}{\alpha}}-1}\right)^{-\alpha} \\
& =\lim _{t \downarrow 0}\left(\frac{(u)^{-\frac{1}{\alpha}}+(v)^{-\frac{1}{\alpha}}}{2}-\frac{1}{2(t)^{-\frac{1}{\alpha}}-1}\right)^{-\alpha} \\
& =2^{\alpha}\left((u)^{-\frac{1}{\alpha}}+(v)^{-\frac{1}{\alpha}}\right)^{-\alpha} .
\end{aligned}
$$

\section{B.1 Figure related calculations}

Followings are calculations embedded with Figure 5.2.

- Recall the result obtained on 4.3.1. For Clayton survival copula with $X$ in the MDA 
of Frechet, we have the asymptotic result of JES as following.

$$
\mathbb{E}\left[X \mid X>\zeta F^{\leftarrow}(q), Y>G^{\leftarrow}(q)\right] \sim F^{\leftarrow}(q)\left(\zeta+\frac{1}{\tau\left(\zeta^{-1 / \gamma}, 1\right)} \int_{\zeta}^{\infty} \tau\left(x^{-1 / \gamma}, 1\right) \mathrm{d} x\right)
$$

For $\alpha=1, \tau(u, v)=2\left((u)^{-1}+(v)^{-1}\right)^{-1}$, then $\tau\left(\zeta^{-1 / \gamma}, 1\right)=2\left(\zeta^{1 / \gamma}+1\right)^{-1}$, and $\tau\left(x^{-1 / \gamma}, 1\right)=2\left(x^{1 / \gamma}+1\right)^{-1}$.

Hence, the asymptotic result of JES is

$$
\begin{aligned}
F^{\leftarrow}(q) & \left(\zeta+\frac{1}{2\left(\zeta^{1 / \gamma}+1\right)^{-1}} \int_{\zeta}^{\infty} 2\left(x^{1 / \gamma}+1\right)^{-1} \mathrm{~d} x\right) \\
& =F^{\leftarrow}(q)\left(\zeta+\left(\zeta^{1 / \gamma}+1\right) \int_{\zeta}^{\infty}\left(x^{1 / \gamma}+1\right)^{-1} \mathrm{~d} x\right) .
\end{aligned}
$$

- Refer to Proposition 10 of Hua and Joe (2014), we have the asymptotic result of MES as below.

$$
\mathbb{E}\left[X \mid Y>F^{\leftarrow}(q)\right] \sim F^{\leftarrow}(q) \int_{0}^{\infty} b\left(x^{-1 / \gamma}, 1\right) \mathrm{d} x
$$

For $\alpha=1, b(u, v)=\left((u)^{-1}+(v)^{-1}\right)^{-1}$, then $b\left(x^{-1 / \gamma}, 1\right)=\left(x^{1 / \gamma}+1\right)^{-1}$.

Substituting the above terms into B.1.2, we have the asymptotic result of MES as

$$
F^{\leftarrow}(q) \int_{0}^{\infty}\left(x^{1 / \gamma}+1\right)^{-1} \mathrm{~d} x
$$

By changing $q$ from 0.985 to 0.999 and specifying corresponding $\gamma$ and $\zeta$, Figure 5.2 is generated.

\section{B.2 Table related calculations}

Followings are calculations related to Table 5.2 and 5.3 . 
- The tail dependence coefficient $\lambda$ of Clayton survival copula is:

$$
\lambda=\lim _{t \downarrow 0} \frac{\widehat{C}(t, t)}{t}=\lim _{t \downarrow 0}\left(\frac{t^{-\frac{1}{\alpha}}+t^{-\frac{1}{\alpha}}-1}{t^{-\frac{1}{\alpha}}}\right)^{-\alpha}=\lim _{t \downarrow 0}\left(2-t^{\frac{1}{\alpha}}\right)^{-\alpha}=2^{-\alpha} .
$$

- While $\zeta=1$, equation B.1.1 is,

$$
F^{\leftarrow}(q)\left(1+2^{\alpha} \int_{1}^{\infty}\left(x^{\frac{1}{\alpha \gamma}}+1\right)^{-\alpha} \mathrm{d} x\right)
$$

These elements are sufficient for us to generate results on Table 5.2 and 5.3. 